\title{
Balancing High Open Circuit Voltage over 1.0 V and High Short Circuit Current in Benzodithiophene-Based Polymer Solar Cells with Low Energy Loss: A Synergistic Effect of Fluorination and Alkylthiolation
}

\author{
Zhengkun Du, Xichang Bao,* Yonghai Li, Deyu Liu, Jiuxing Wang, Chunming Yang, \\ Reinhard Wimmer, Lars Wagner Städe, Renqiang Yang,* and Donghong Yu*
}

Based on the most recently significant progress within the last one year in organic photovoltaic research from either alkylthiolation or fluorination on benzo[1,2-b:4,5- $\left.b^{\prime}\right]$ dithiophene moiety for high efficiency polymer solar cells (PSCs), two novel simultaneously fluorinated and alkylthiolated benzo[1,2-b:4,5- $\left.b^{\prime}\right]$ dithiophene (BDT)-based donor-acceptor (D-A) polymers, poly(4,8-bis(5'-((2"-ethylhexyl)thio)-4'-fluorothiophen-2'-yl) benzo[1,2-b:4,5- $\left.b^{\prime}\right]$ dithiophene-2,6-diyl)-alt-2'-ethylhexyl-3-fluorothieno[3,4-b] thiophene-2-carboxylate (PBDTT-SF-TT) and poly(4,8-bis (5'-( $2^{\prime \prime}$-ethylhexyl) thio)-4'-fluorothiophen-2'-yl)benzo[1,2-b:4,5-b']dithiophene-2,6-diyl)-alt-1,3bis(thiophen-2-yl)-5,7-bis(2-ethylhexyl)benzo[1,2-c:4,5-c' $]$ dithiophene-4,8-dione (PBDTT-SF-BDD), namely, via an advantageous and synthetically economic route for the key monomer are reported herein. Synergistic effects of fluorination and alkylthiolation on BDT moieties are discussed in detail, which is based on the superior balance between high $V_{o c}$ and large $J_{s c}$ when PBDTTSF-TT/PC 71 BM and PBDTT-SF-BDD/PC $C_{71} B M$ solar cells present their high $V_{\text {oc }}$ as 1.00 and $0.97 \mathrm{~V}$ (associated with their deep highest occupied molecular orbital level of -5.54 and $-5.61 \mathrm{eV}$ ), a moderately high $J_{\mathrm{sc}}$ of 14.79 and $14.70 \mathrm{~mA} \mathrm{~cm}^{-2}$, and thus result a high power conversion efficiency of $9.07 \%$ and $9.72 \%$, respectively. Meanwhile, for PBDTT-SF-TT, a very low energy loss of $0.59 \mathrm{eV}$ is pronounced, leading to the promisingly high voltage, and furthermore performance study and morphological results declare an additivefree PSC from PBDTT-SF-TT, which is beneficial to practical applications. from over $11 \%^{[1,2]}$ and $12 \%[3]$ up to over $13 \%$ now $^{[4]}$ in either tandem or single junction solar cells from benzo[1,2-b:4,5$b^{\prime}$ ] dithiophene (BDT)-based polymers as the donor material in photovoltaic (PV) devices, their limiting open-circuit voltage $\left(V_{\mathrm{oc}}\right.$, below $\left.1.00 \mathrm{~V}\right)$ is still one of the key obstacles to achieve ideally high PV performance with minimized trade-off on another characteristic features of the OPV devices, i.e., the short-circuit current density $\left(U_{\mathrm{sc}}\right)$. For the first two single junction polymer solar cells (PSCs) with PBDB-T/3, 9-bis (2-methylene-(3-(1,1dicyanomethylene)-indanone) )-5,5,11,11tetrakis(4-hexylphenyl)-dithieno[2,3-d:2',3' $\mathrm{d}^{\prime}$ ]-s-indaceno[1,2-b:5,6-b']dithiophene (ITIC) and PBDT-T/IT-M as active layer materials, respectively, their photon energy loss (defined as $E_{\mathrm{Loss}}=E_{\mathrm{g}}-e V_{\mathrm{oc}},{ }^{[5,6]}$ whereas $E_{\mathrm{g}}$ refers to the band gap energy difference between the energy level of highest occupied molecular orbital (HOMO) and lowest unoccupied molecular orbital (LUMO)) has been pronounced as 0.66 and $0.69 \mathrm{eV}$, respectively, which is still above the empirically optimized level of $0.60 \mathrm{eV} .^{[7]}$ Despite their breaking PCE values, these nonideally low $E_{\text {Loss }}$ still reflect the imperfect molec-

\section{Introduction}

Although just within the last one year the power conversion efficiency (PCE) of organic photovoltaics (OPVs) has been boosted

Dr. Z. Du, Prof. R. Wimmer, Dr. L. W. Städe, Prof. D. Yu

Department of Chemistry and Bioscience

Aalborg University

DK-9220 Aalborg, Denmark

E-mail: yu@bio.aau.dk

Dr. Z. Du, Prof. D. Yu

Sino-Danish Center for Education and Research (SDC)

Niels Jensens Vej 2, DK-8000 Aarhus, Denmark

The ORCID identification number(s) for the author(s) of this article can be found under https://doi.org/10.1002/aenm.201701471. ular design of the PBDB-T backbone structure for ideal control on their HOMO/LUMO levels. $E_{\mathrm{Loss}}, E_{\mathrm{g}}, V_{\mathrm{oc}}$, and $J_{\mathrm{sc}}$ for [6,6]-phenyl$\mathrm{C}_{71}$-butyric acid methyl ester $\left(\mathrm{PC}_{71} \mathrm{BM}\right) / \mathrm{PSCs}$ from BDT and/ or 2'-ethylhexyl-3-fluorothieno[3,4-b]thiophene-2-carboxylate (TT,

DOI: 10.1002/aenm.201701471 

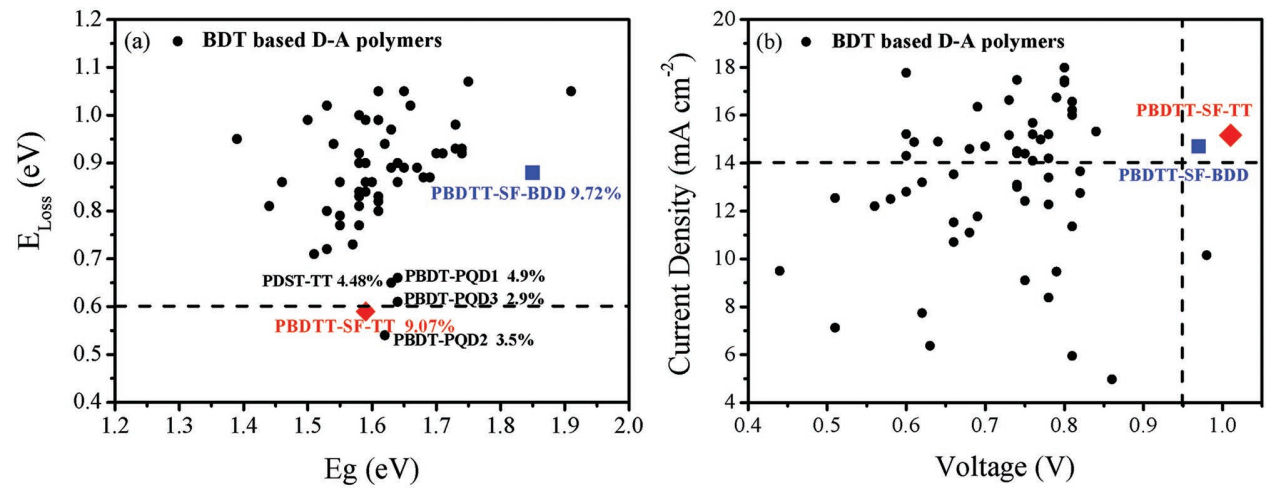

Figure 1. a) Correlation between energy loss and band gap for PBDTT-SF-TT, PBDTT-SF-BDD, and BDT and/or TT-based D-A polymers from literature. b) $J_{\mathrm{Sc}}$ and $V_{\mathrm{oc}}$ for PBDTT-SF-TT, PBDTT-SF-BDD, and BDT and/or TT-based D-A polymers from literature. Black spots: literature reported BDT and/or TT-based D-A polymers, red diamond and blue square: PBDTT-SF-TT and PBDTT-SF-BDD.

fluorinated and carboxylated)-based donor-acceptor (D-A) polymers have been summarized in Figure 1a,b and Table S1 in the Supporting Information, respectively. To date, only one polymer PBDT-PQD2 ${ }^{[8]}$ and other three polymers PBDT-PQD1, PBDTPQD3, ${ }^{[8]}$ and PDST-TT are found in $E_{\text {Loss }}$ level below or close to $0.6 \mathrm{eV}$ (the dash line in Figure 1a), while their low PCEs have been totally out from the scope of state-of-the-art OPV development. Meanwhile, prior to the fill factor (FF), coordinately high $V_{\mathrm{oc}}$ and $J_{\mathrm{sc}}$ should be expected; however, in BDT-polymer/ fullerene solar cells as illustrated in Figure 1b, the gain of large $J_{\mathrm{sc}}$ and high $V_{\mathrm{oc}}$ are in fact contradictory: for polymer $/ \mathrm{PC}_{71} \mathrm{BM}$ cells, only one of the polymers, PDST-TT ${ }^{[9]}$ attains a $V_{\text {oc }}>0.90 \mathrm{~V}$, while its $J_{\mathrm{sc}}$ only remains at $10.15 \mathrm{~mA} \mathrm{~cm}^{-2}$; for other polymers (e.g., PBT-3F, ${ }^{[10]}$ PBDT-TS1, ${ }^{[11]}$ PBDT-TSR ${ }^{[12]}$ ) whose $J_{\mathrm{Sc}} \mathrm{S}$ are higher than $14.00 \mathrm{~mA} \mathrm{~cm}^{-2}$, their $V_{\text {oc }}$ all fall below $0.86 \mathrm{~V}$, few of them stay in between 0.80 and $0.86 \mathrm{~V}$, even though their PCE could have reached high values as $8.6 \%, 9.48 \%$, and $10.20 \%$.

To date, rational design rules on molecular level for exquisite control on simultaneous low $E_{\text {Loss }} /$ high $V_{\text {oc }}$ or high $V_{\text {oc }} /$ large $J_{\text {sc }}$ are still ambiguous. First, owing to its balanced weak electron donating nature and moderate $\pi$-electron accepting capability into its 3d-orbitals at the aryl or allyl position, ${ }^{[13]}$ alkylthiol group had been pioneer into poly[5-methoxy-2-(2'-ethyl-hexyloxy)-pphenylenevinylene] (MEH-PPV) by Hou et al. ${ }^{[14]}$ for effectively tuning its HOMO to deeper levels. Later alkylthiolation on 1D BDT by Ferraris and co-workers ${ }^{[15,16]}$ and on 2D-conjugated BDT moiety by Li and co-workers ${ }^{[17]}$ (PBDTT-S-TT) and Hou and co-workers ${ }^{[1]}$ (PBDT-TS1) had been sparkled as effective design strategy on D-A polymer for low-lying HOMO levels and therefore improved $V_{\text {oc }}$ as 0.84 and $0.80 \mathrm{~V}$, resulting their PCE of $8.42 \%$ and $9.48 \%$. Second, relying on its small van der Waals radius and high electronegativity (3.98 on the Pauling scale), ${ }^{[18]}$ fluorine $(\mathrm{F})$ atom favors $\mathrm{F}-\pi, \mathrm{F}-\mathrm{H}$, and $\mathrm{F}-\mathrm{S}^{[19]}$ inter- and intramolecular interactions and therefore fluorination would simultaneously increase both the electron affinity (EA) and ionization potential (IP) of the conjugated polymers with a deepening of both LUMO and HOMO without significantly affecting the $E_{\mathrm{g}}{ }^{[20-24]}$ Nevertheless, despite that most of these work focused on fluorination on the acceptor moiety (TT-ester, for example) in the D-A polymers, $\mathrm{Yu}$ and co-workers and Hou and co-workers systematically studied the multifluorination on both $\mathrm{D}$ and $\mathrm{A}$ moieties and proved that introduction of $\mathrm{F}$ atom(s) into various positions at alkyl-, alkylthieonyl-BDT, and TT motifs (PTBFx series ${ }^{[20]}$ and PBT- $\left.x \mathrm{~F}^{[10]}(x: 0-3)\right)$ led to distinct influences on their PV performance. For the former, HOMO level had been systematically lowered from $-4.94 \mathrm{eV}$ for PTBF0 to $-5.48 \mathrm{eV}$ for PTBF3; for the latter, they started deepening HOMO from $-4.73 \mathrm{eV}$ for PBT-0F to $-4.90 \mathrm{eV}$ for PBT-3F, corresponding to the voltage elevation from 0.58 to $0.75 \mathrm{~V}$ and from 0.56 to $0.78 \mathrm{~V}$, respectively.

Despite the above-introduced two effective methods of alkylthiolation or fluorination on BDT moiety for fine-tuning HOMO/LUMO levels and therefore indeed increased $V_{\mathrm{oc}}$, losses of $J_{\mathrm{sc}}$ had been always found, as what one can recall in Figure 1b. Until now, no combination of these two as design idea has been merged. Therefore, in order to investigate the synergistic effects of alkylthiolation and fluorination on controls of the levels of molecular orbitals, in this contribution, we report two novel simultaneously alkylthiolated and fluorinated BDT-based D-A polymers: poly(4,8-bis(5'-((2"-ethylhexyl)thio)$4^{\prime}$-fluorothiophen-2'-yl)benzo[1,2-b:4,5- $\left.b^{\prime}\right]$ dithiophene-2,6-diyl)alt-2'-ethylhexyl-3-fluorothieno[3,4-b]thiophene-2-carboxylate (PBDTT-SF-TT) and poly(4,8-bis(5'-((2" -ethylhexyl)thio)-4'fluorothiophen-2'-yl)benzo[1,2- $\left.b: 4,5-b^{\prime}\right]$ dithiophene-2,6-diyl)alt-1,3-bis(thiophen-2-yl)-5,7-bis(2-ethylhexyl)benzo[1,2-c:4,5-c'] dithiophene-4,8-dione (PBDTT-SF-BDD), which combines alkylthiolation and fluorination on BDT motif as electrondonor moiety and TT or BDD as electron-deficient unit for constructing D-A polymers.

As shown in Scheme 1, the key monomer 4,8-bis(5'-((2"ethylhexyl)thio)-4'-fluorothiophen-2'-yl)benzo[1,2-b:4,5- $\left.b^{\prime}\right]$ dithiophene-2,6-diyl)-bis(trimethylstannane) (M1, BDTT-SF) was synthesized within three steps of reaction with reasonably high yield, which was polymerized via Stille coupling reactions with dibromo-compounds of TT and BDD, respectively. The chemical structure, thermal stability, electrochemical- and photophysical-properties of PBDTT-SF-TT and PBDTT-SF-BDD were investigated. The $\mathrm{PC}_{71} \mathrm{BM}$-based fullerene OPV devices for both PBDTT-SF-TT and PBDTT-SF-BDD were fabricated, their PV performance, hole mobility, and thin film morphology were well studied. The synergistic effects of alkylthiolation and fluorination on energy losses, band gap, $V_{\mathrm{oc}}, J_{\mathrm{sc}}$, and final PCE were discussed in detail. 


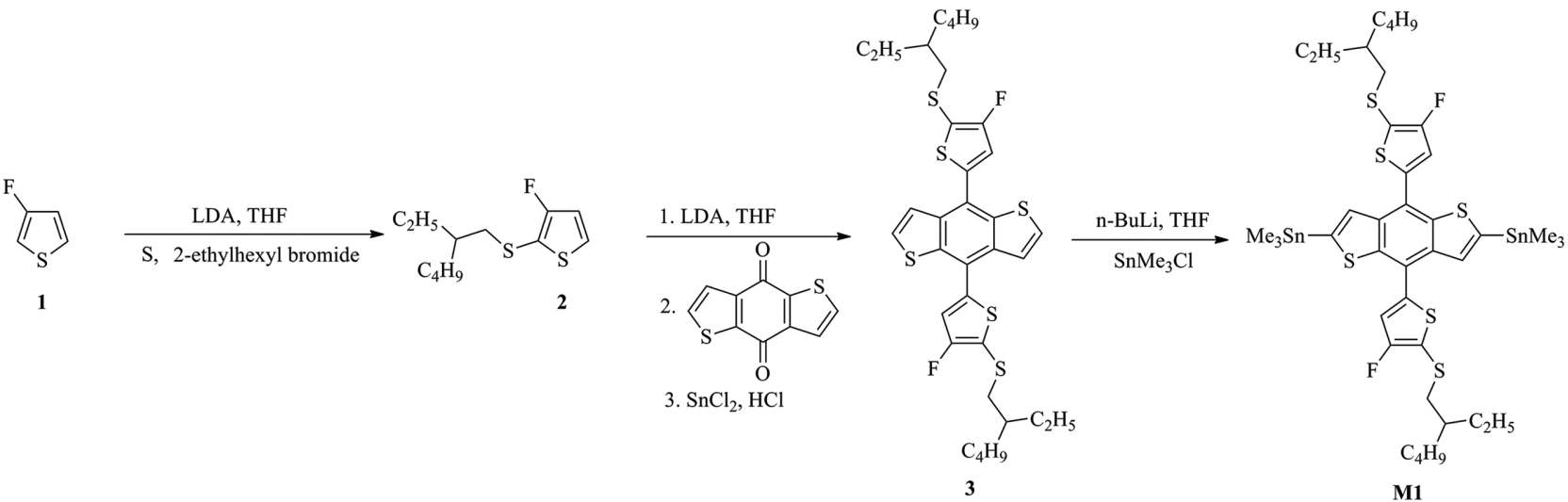

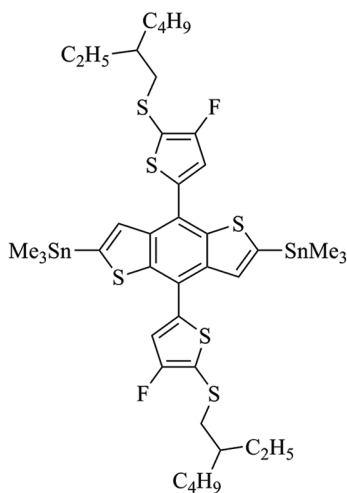

M1

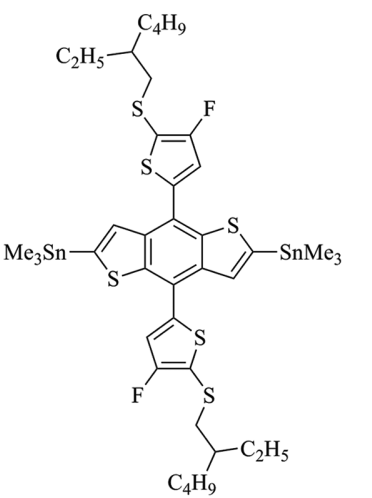

M1

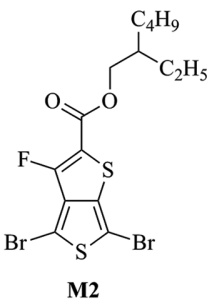

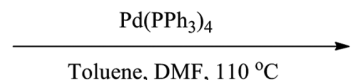

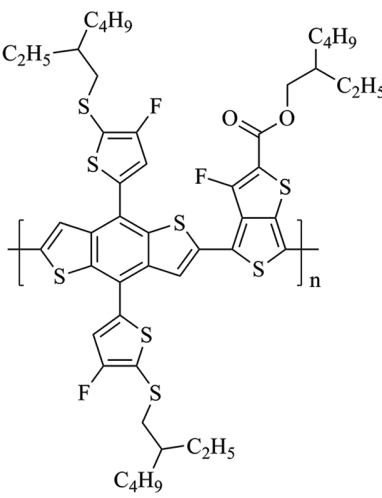

PBDTT-SF-TT

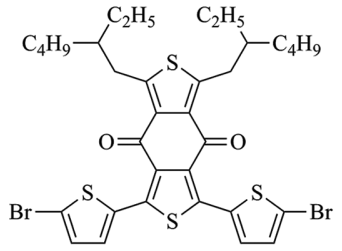

BDD

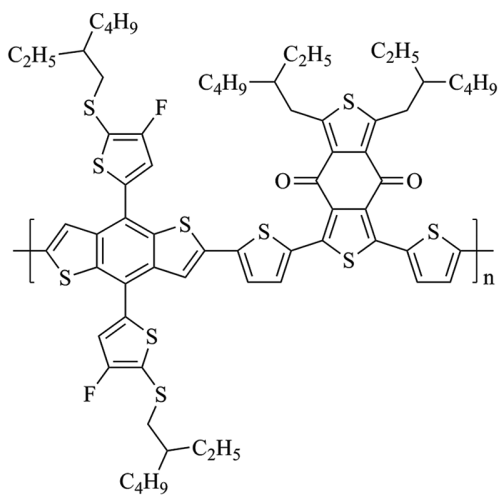

PBDTT-SF-BDD

Scheme 1. Chemical structures and synthetic routes of monomer M1 (bis-stannylated-(fluorinated-alkylthiophenyl-BDT), namely, BDTT-SF) and the target polymer PBDTT-SF-TT and PBDTT-SF-BDD.

\section{Results and Discussion}

\subsection{Syntheses and Characterization}

The detailed synthetic procedure of (4,8-bis(5'-(2"-ethylhexyl) thio- $4^{\prime}$-fluorothiophen-2'-yl) benzo[1,2-b:4,5- $\left.b^{\prime}\right]$ dithiophene2,6-diyl)bis(trimethylstannane) (M1, BDTT-SF) were given in the Experimental Section. As the three synthetic steps illustrated in Scheme 1, such a synthetic route avoided the synthetically unfavored trimethylsilyl (TMS) protection and deprotection procedure, appearing more sound when comparing to the synthesis of similar monomer (fluorinated alkylthieonyl BDT) by Hou and co-workers ${ }^{[10]}$ The resulted key monomer M1
(BDTT-SF) was fully characterized by ${ }^{1} \mathrm{H}$ NMR, ${ }^{13} \mathrm{C} N M R$, and matrix assisted laser desorption/ionization time of flight mass spectrometry (MALDI-TOF MS). To obtain the target polymer PBDTT-SF-TT and PBDTT-SF-BDD, the polymerization was carried out by a $\mathrm{Pd}\left(\mathrm{PPh}_{3}\right)_{4}$-catalyzed Stille coupling reaction between monomer M1 (BDTT-SF) and 2'-ethylhexyl4,6-dibromo-3-fluorothieno[3,4-b]thiophene-2-carboxylate (M2, TT) and 1,3-bis(5-bromothiophen-2-yl)-5,7-bis(2-ethylhexyl) benzo[1,2-c:4,5-c']dithiophene-4,8-dione (BDD) in refluxed mixture of toluene and $N, N$-dimethylformamide (DMF) under nitrogen. The size exclusion chromatography (SEC) using chloroform $\left(\mathrm{CHCl}_{3}\right)$ as the eluent pronounces a number-average molecular weight $\left(M_{n}\right)$ of 58127 Da with a polydispersity index 
(PDI) of 2.53 for PBDTT-SF-TT and $M_{n}$ of 33400 Da with a PDI of 3.92 for PBDTTT-SF-BDD, respectively. Furthermore, the thermogravimetric analysis (TGA) plot of PBDTT-SF-TT and PBDTT-SF-BDD as shown in Figure S1 in the Supporting Information reveals an onset decomposition temperature at $5 \%$ weight loss $\left(T_{\mathrm{d}}\right)$ occurring at $\approx 328$ and $334{ }^{\circ} \mathrm{C}$, whose former is higher than that of only alkylthiolated PBDT'T-S-TT $\left(291{ }^{\circ} \mathrm{C}\right)$ as reported by $\mathrm{Li}$ and co-workers ${ }^{[17,25]}$ This evidences sufficient thermal stabilities for PBDTT-SF-TT-based PSC applications.

\subsection{Optical Properties}

The UV-vis absorption spectra of PBDTT-SF-TT and PBDTTSF-BDD were acquired in both dilute chlorobenzene (CB) solution and film at ambient and elevated temperatures, as found in Figure 2. The related data are collected in Table 1. From solution to film, PBDTT-SF-TT and PBDTT-SF-BDD both exhibited comparable broad and strong absorption in the region from $300 \mathrm{~nm}$ to $\approx 780 \mathrm{~nm}$, without a commonly visible bathochromic shift of its maximum absorption, implying effective aggregation of these two polymer chains formed in solution phase. This could be attributed to the strong intermolecular interactions resulted from the simultaneous fluorination effect on both the BDT donor unit and TT acceptor moiety on the backbone, which can cause $\mathrm{F}-\mathrm{H}$ and $\mathrm{F}-\mathrm{S}$ interactions. ${ }^{[19,26]}$ Comparing with the weak absorption for the $\pi-\pi^{*}$ transition below $400 \mathrm{~nm}$, the intense absorption peak at $640 \mathrm{~nm}$ in solution, arising from the intramolecular charge transfer (ICT) between BDT (as donor) and TT and BDD (as acceptor) moieties, indicates a strong electron-withdrawing effects caused by fluorinated TT-ester and BDD. The strong intermolecular $\pi-\pi$ stacking presented as the absorption peak at $700 \mathrm{~nm}$ for PBDTT-SFTT and $617 \mathrm{~nm}$ for PBDTT-SF-BDD can be expected to facilitate intermolecular charge transport, which has been explored in many OPV polymers. ${ }^{[17,28-30]}$ Such strong $\pi-\pi$ stacking has been also experimentally evidenced by their very poor solubility in $\mathrm{CB}$ or o-dichlorobenzene (o-DCB) at room temperature even though its molecular weight is moderately high. This is furthermore pronounced by the higher peak absorbance ratio between the absorption of $\pi-\pi$ stacking band and ICT band for PBDTT-SF-TT and PBDTT-SF-BDD when comparing them with PBDTT-S-TT, ${ }^{[17]}$ PBT-3F, ${ }^{[10]}$ which again suggests a synergistic effect of fluorination and alkylthiolation of BDT moiety on their improved molecular aggregation. Comparing the neither alkylthiolated nor fluorinated control polymer PBDTT-TT/ PBT-1F (the two identical polymer backbones) with PBDTTS-TT and with PBT-3F, their respective bathochromic- and blueshifts of the peak adsorption of $\pi-\pi$ stacking band in film as found in Table 1 clearly evidenced a contradictory effect arosen from sole alkylthiolation and mere fluorination, while our simultaneous alkylthiolation and fluorination just coordinately led to such a balanced aggregation when seeing that of PBDTT-SF-TT (700 nm) lying in between that of only alkylthiolated PBDTT-S-TT (716 nm) and only fluorinated PBT-3F $(665 \mathrm{~nm})$. Besides these, it is also noticeable that the absorption edge of PBDTT-SF-TT film is $16 \mathrm{~nm}$ blueshifted comparing to PBDTT-S-TT, but $35 \mathrm{~nm}$ redshifted when referring to PBT-3F, which implies that alkylthiolation of thiophene motif might have deepened more the HOMO level than fluorination does,
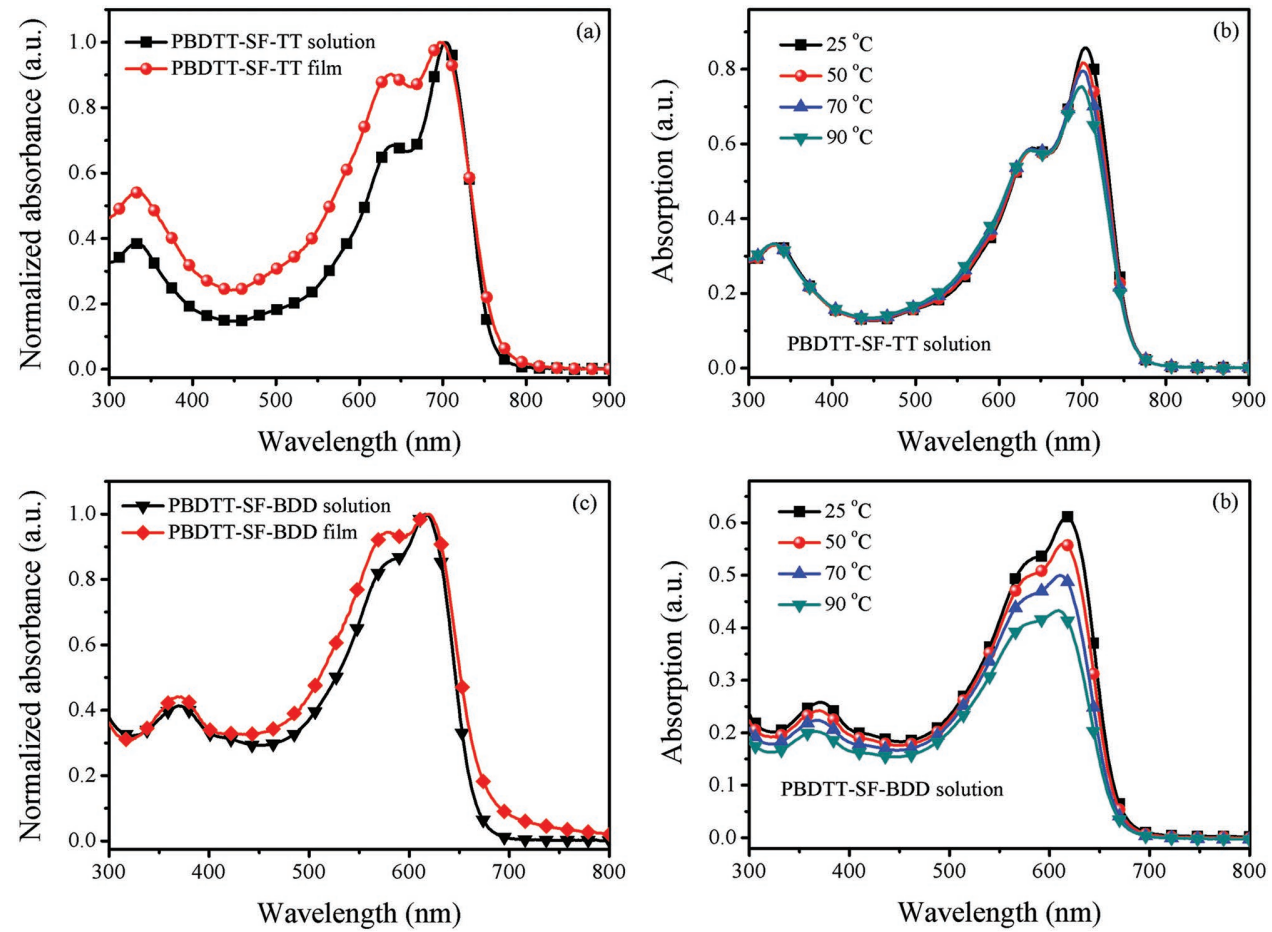

Figure 2. UV-vis absorption spectra of PBDTT-SF-TT and PBDTT-SF-BDD: a) PBDTT-SF-TT in chlorobenzene solution and thin film on quartz at ambient temperature, b) temperature-dependent absorption of PBDTT-SF-TT in solution, c) PBDTT-SF-BDD in chlorobenzene solution and thin film on quartz at ambient temperature, d) temperature-dependent absorption of PBDTT-SF-BDD in solution. 
Table 1. Photophysical and electrochemical parameters of PBDTT-SF-TT, PBDTT-SF-BDD, and reference polymers from literature.

\begin{tabular}{|c|c|c|c|c|c|c|c|c|}
\hline \multirow[t]{2}{*}{ Polymer } & \multicolumn{2}{|c|}{$\begin{array}{l}\lambda_{\max } \\
{[\mathrm{nm}]}\end{array}$} & \multirow[t]{2}{*}{$\begin{array}{l}\lambda_{\text {edge }} \\
{[\mathrm{nm}]}\end{array}$} & \multirow[t]{2}{*}{$\begin{array}{c}\mathrm{HOMO}^{\mathrm{a})} \\
{[\mathrm{eV}]}\end{array}$} & \multirow[t]{2}{*}{$\begin{array}{c}\text { LUMO } \\
{[\mathrm{eV}]}\end{array}$} & \multirow[t]{2}{*}{$\begin{array}{c}\mathrm{HOMO}^{\mathrm{b})} \\
{[\mathrm{eV}]}\end{array}$} & \multirow[t]{2}{*}{$\begin{array}{c}\text { LUMOb) } \\
{[\mathrm{eV}]}\end{array}$} & \multirow[t]{2}{*}{$\begin{array}{c}\left.E_{\mathrm{g}}^{\mathrm{optc}}\right) \\
{[\mathrm{eV}]}\end{array}$} \\
\hline & Solution & Film & & & & & & \\
\hline PBDTT-SF-TT & 640,703 & 638,700 & 780 & -5.54 & $-3.95^{\mathrm{d})}$ & -5.23 & -2.88 & 1.59 \\
\hline PBDTT-S-TT & 644,709 & 657,716 & 790 & -5.41 & -3.27 & -5.22 & -2.77 & $1.57^{[17]}$ \\
\hline PBDTT-TT') & 640,708 & 643,705 & 784 & -5.30 & -3.17 & -5.04 & -2.66 & $1.58^{[17]}$ \\
\hline PBT-3F & & 612,665 & 755 & -5.20 & -3.30 & -4.90 & -2.75 & $1.64^{[10]}$ \\
\hline PBT-1 Fe) & & 620,673 & 750 & -4.95 & -3.12 & -4.78 & -2.64 & $1.65^{[10]}$ \\
\hline PBDTT-SF-BDD & 580,617 & 578,617 & 671 & -5.61 & $-3.77^{\mathrm{d})}$ & -5.09 & -2.69 & 1.85 \\
\hline PBI-S & & 621,630 & 696 & -5.30 & $-3.52^{\mathrm{d})}$ & & & $1.78^{[27]}$ \\
\hline
\end{tabular}

a) Measured by cyclic voltammetry; ${ }^{\text {b) }}$ Calculated from DFT; ${ }^{c}$ )Evaluated from the absorption band edge of the polymer film; ${ }^{\text {d) }}$ Calculated from the equation $E_{L u m O}=E_{H O M O}+$ $E_{\mathrm{g}}{ }^{\text {opt; }}$ e) Completely identical polymer backbone structure between PBT-1F and PBDTT-TT as structural-control to exclusively fluorinated and solely alkylthiolated polymers by $\mathrm{Hou}$ and co-workers ${ }^{[10]}$ and $\mathrm{Li}$ and co-workers, ${ }^{[17]}$ respectively.

therefore resulting a slightly wider band gap than PBDTT-S-TT and lower band gap than PBT-3F. The optical band gap $\left(E_{\mathrm{g}}{ }^{\mathrm{opt}}\right)$ of PBDTT-SF-TT and PBDTT-SF-BDD based on the optical absorption edges of the film spectra is 1.59 and $1.84 \mathrm{eV}$, respectively. For PBDTT-SF-TT, its bandgap is slightly larger than those of polymers with the similar structure implying a larger $V_{\text {oc }}$ could be expected for PBDTT-SF-TT-based PSCs. From the optical absorption characteristics between PBDTT-SF-BDD and its structural analog PB1-S ${ }^{[27]}$ which was only alkylthiolated, further fluorination on it in fact presented a result of weakened electron "push-pull" effect between BDT-TT and BDD units, again reflecting the synergistic effect of fluorination and alkylthiolation as just above-discussed in PBDTT-SF-TT versus PBDTT-S-TT. Therefore its larger bandgap than PB1-S' could be resulted from the higher LUMO level due to both this interactive fluorination and akylthiolation on BDTT motif and the lack of fluorination on acceptor unit.

The temperature dependence on macromolecular aggregation via UV-vis absorption study on both PBDTT-SF-TT and PBDTT-SF-BDD was investigated in dilute chlorobenzene solution under different temperature, as illustrated in Figure $2 b, d$. These spectra demonstrate remarkable blueshifts, slightly for PBDTT-SF-TT but dramatically for PBDTT-SF-BDD, declined absorption intensity, and narrowing of the absorption bands as the solution temperature arises, revealing dissociation of aggregated PBDTT-SF-TT and PBDTT-SF-BDD. Despite at the high temperature (up to $90{ }^{\circ} \mathrm{C}$ ), the solution still presents the distinct and sharp shoulder peak at the long wavelength region, indicating much stronger aggregation of both PBDTT-SF-TT and PBDTT-SF-BDD than those of polymers with the similar structures. ${ }^{[10,17,28,30-33]}$

\subsection{Electrochemical Properties}

The electrochemical characteristics of PBDTT-SF-TT and PBDTT-SF-BDD in thin films were studied by means of cyclic voltammetry $(\mathrm{CV})$ as detail-instructed in the Experimental Section. The HOMO energy level of PBDTT-SF-TT, PBDTT-SFBDD, and those of literature reported PBDTT-S-TT and PBT-3F are listed together with their optical performance in Table 1 (including the theoretically calculated results). The experimental results have the same tendency as those computersimulated when comparing with their nonfluorinated structural analogues PBDTT-S-TT and PB1-S. As exhibited in Figure 3, PBDTT-SF-TT and PBDTT-SF-BDD showed one reversible oxidation process. The oxidation onset found at $1.13 \mathrm{~V}$ for PBDTTSF-TT and $1.20 \mathrm{~V}$ for PBDTT-SF-BDD resulted in an IP of 5.54 and $5.61 \mathrm{eV}$, suggesting the HOMO level of $-5.54 \mathrm{eV}$ for PBDTT-SF-TT and $-5.61 \mathrm{eV}$ for PBDTT-SF-BDD. For PBDTTSF-TT, its HOMO level proclaims $0.13 \mathrm{eV}$ lower than that of PBDTT-S-TT and $0.34 \mathrm{eV}$ lower than that of PBT-3F, which are their structural counterparts. While for PBDTT-SF-BDD, comparing with its nonfluorinated analogue PB1-S, ${ }^{[27]}$ the difference is also as large as $0.31 \mathrm{eV}$. Such more deepened HOMO level proved the strong induction effect caused by fluorination on alkylthiolated BDTT, endowing its high EA, and also high oxidation stability which is desirable for application in devices, especially when referring to the sole structural difference between these two BDTT-based 2D-conjugated polymers. As agreed in OPV community, introduction of $\mathrm{F}$ atoms on acceptor moiety would lead to reduced HOMO and LUMO at the same time, from the above presented, further including $\mathrm{F}$ atom on

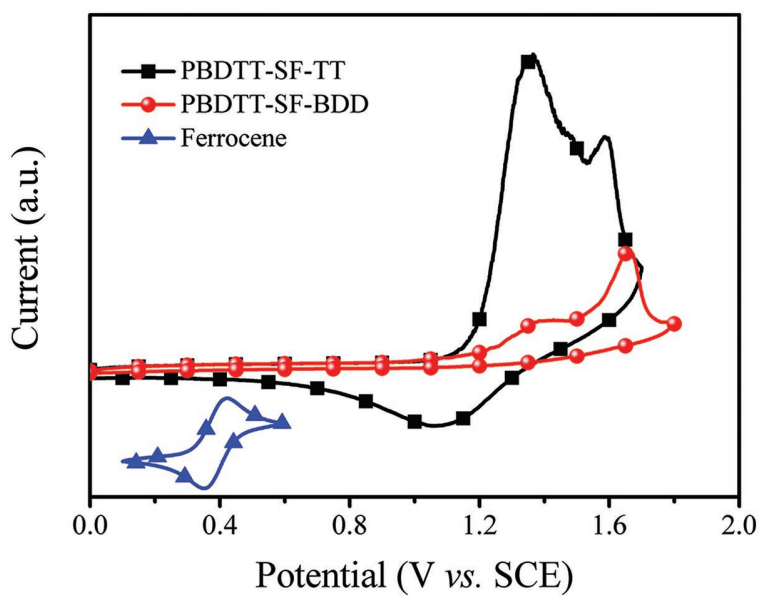

Figure 3. Cyclic voltammograms of PBDTT-SF-TT, PBDTT-SF-BDD, and $\mathrm{Fc} / \mathrm{Fc}^{+}$. 
already alkylthiolated donor motif (from PBDTT-S-TT ${ }^{[17]}$ to PBDTT-SF-TT) could still keep decreasing their HOMO levels; however, not as significantly as alkylthiolation did on already fluorinated ones (from PBT-3F ${ }^{[10]}$ to PBDDTT-SF-TT), which had been also reflected by again the coordinative effect of $-F$ and -SR bonding in the BDTT donor unit as just discussed on their photophysical properties. Meanwhile, the decreasing HOMO level from the barely ethylhexylthiolated analog PB1-S to PBDTT-SF-BDD pronounced as significantly as that from its alkylthiolated analog PBDTT-S-TT to PBDTT-SF-TT because of missing $\mathrm{C}-\mathrm{F}$ bonding in the BDD acceptor (comparing to TT). Due to the lack of well-defined reduction, neither EA nor related LUMO energy level could be derived. In spite of this, combining the HOMO levels with the $E_{\mathrm{g}}{ }^{\text {opt }}$ obtained from its optical absorption affords a LUMO level of $-3.95 \mathrm{eV}$ for PBDTT-SF-TT and $-3.77 \mathrm{eV}$ for PBDTT-SF-BDD.

\subsection{Molecular Simulations}

Molecular simulation (density-functional theory (DFT) calculations) on the dimer of BDTT-SF-TT and BDTT-SF-BDD were conducted by using Gaussian 09 program with B3LYP functional at the $6-31 \mathrm{G}(\mathrm{d}, \mathrm{p})$ basis set level in the gas phase. All the alkylthiol side chains were replaced by methylthiol groups to reduce the computational load of the calculations. As one can see in Figure S2a,b, Supporting Information, the planarity of the backbone in both PBDTT-SF-TT and PBDTT-SF-BDD are evidenced, due to that the promising dihedral angles within D-A-D-A (between the BDT motif and TT and BDD), being $0.93^{\circ}, 20.10^{\circ}, 28.80^{\circ}$ and $5.37^{\circ}, 13.47^{\circ}, 10.18^{\circ}$, respectively, confess the twisting and a certain coil-like polymer conformation as a result of enhanced torsional relaxation along the backbone. ${ }^{[34]}$ This can, to some extent, be indicated by the none visible bathochromic shift on the absorption maxima between the solutions and films for either PBDTT-SF-TT or PBDTT-SF-BDD as already discussed above. Furthermore, the dihedral angles of $55^{\circ}-61^{\circ}$ and $52^{\circ}-57^{\circ}$ between the fluorinated-alkylthiolated thiophene (T-SF) unit and BDT moiety prevent the overall planarity of the polymer, which already existed as the torsion angle of $57.1^{\circ}$ between the exclusively alkylthiolated thiophene side unit and BDT motif (termed as PBT-TS). ${ }^{[35]}$ This indicates that neither induction nor steric effect caused by side-chain fluorination on BDT could improve the intramolecular planarity in the donor segments of the backbone, while only fluorination on the main chain (directly on BDT) can straightforwardly influences the planarity between the D and A motifs as reported by $\mathrm{Yu}$ and co-workers ${ }^{[20]}$ This is expected to have a negative influence on the band gap properties, as the spatial steric effect will affect the $\pi-\pi$ stacking intermolecularly. It is important to mention that all side-chain substituents were replaced with a methylthiol group in order to simplify the calculations; nevertheless, the side chains typically play an important role in modeling torsion angels of conjugated polymers. ${ }^{[36]}$ Herein we used the same simplified side group for both moieties of BDT and TT and BDT and BDD, therefore the results regarding to the torsion angles between $\mathrm{BDT}$ and TT/BDD should be reasonable. When observing the orbital distribution (Figure 4), it nonsurprisingly becomes

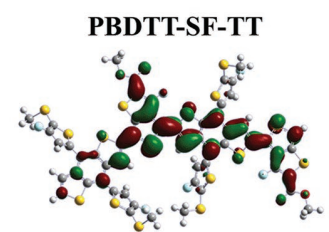

LUMO $=-2.88 \mathrm{eV}$
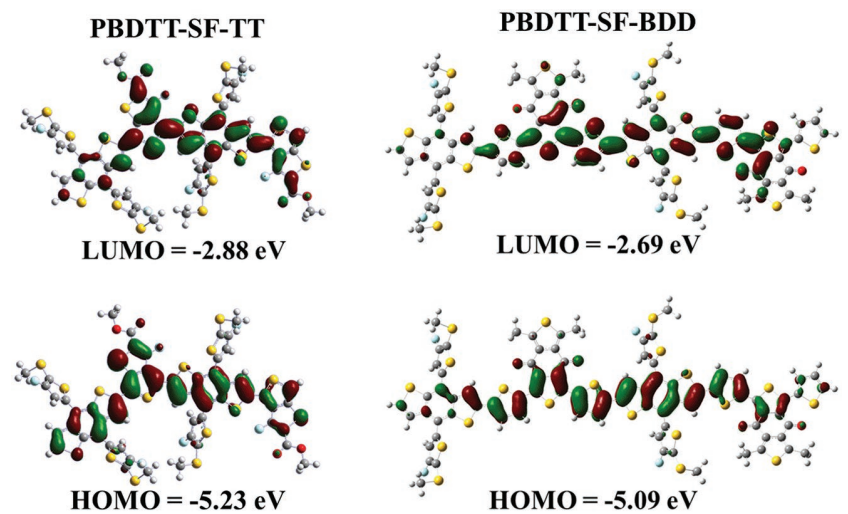

Figure 4. Theoretical calculations (DFT) on PBDTT-SF-TT and PBDTT-SF-BDD.

evident that HOMO covers more in the BDT donor units while LUMO occupies more in TT/BDD acceptor motifs. However, it is noticeable that in both molecules, both HOMO and LUMO migrate into fluorinated thiophene ring in BDT, strongly evidencing the induction effect from $\mathrm{F}$ atom on simultaneously lowering HOMO/LUMO level of the two polymers, when comparing to their structural analogues such as PBDTT-STT and PB1-S. This further supports our discussion on their optical absorptions regarding their moderately expanded band gap when referring to those of PBDTT-S-TT and PB1-S, indicating the lower electron rich properties of the core BDT unit in the backbone, which also improves the electron affinity of the orbitals and leads to a possible deeper HOMO level: the experimental $-5.54 \mathrm{eV}$ for PBDTT-SF-TT and $-5.61 \mathrm{eV}$ for PBDTT-SF-BDD are all deeper than -5.41 and $-5.20 \mathrm{eV}$ for PBDTT-S-TT and PBT-3F and $-5.30 \mathrm{eV}$ for PB1-S. Meanwhile, Li and co-workers ${ }^{[17]}$ and Hou and co-workers ${ }^{[10]}$ have already respectively claimed the HOMO level enhancement: from -5.30 to $-5.41 \mathrm{eV}$ by just alkylthiolation on PBDTT-TT leading to PBDTT-S-TT and from -4.85 to $-5.20 \mathrm{eV}$ by only fluorination on PBT-1F resulting PBT-3F (found in Table 1). Our results clearly demonstrate that it is the synergistic effect of fluorination and alkylthiolation on BDT lowering the low lying HOMO level that leads to high $V_{\text {oc }}$ of 1.00 and $0.97 \mathrm{~V}$ (presented below) for solar cells made from PBDTT-SF-TT and PBDTT-SF-BDD.

\subsection{Photovoltaic Performance of Fullerene-Based Solar Cells}

Fullerene-based $\left(\mathrm{PC}_{71} \mathrm{BM}\right)$ bulk heterojunction (BHJ) OPVs were fabricated using PBDTT-SF-TT or PBDTT-SF-BDD as the donor and $\mathrm{PC}_{71} \mathrm{BM}$ as the acceptor under different mass ratios. The device structure applied was ITO/PEDOT: PSS/Polymer: $\mathrm{PC}_{71} \mathrm{BM} / \mathrm{PFN} / \mathrm{Al}$, where ITO is indium tin oxide, PEDOT: PSS is poly(3,4-ethylenedioxythiophene):polystyrene sulfonate, PFN is poly[(9,9-bis(3'-(N,N-dimethylamino)propyl)-2,7-fluorene)-alt-2,7(9,9-ioctylfluorene)]. $J-V$ and external quantum efficiencies (EQE) curves for both PBDTT-SF-TT: PC $_{71} \mathrm{BM}$ (1:1.5) and PBDTT-SFBDD: $\mathrm{PC}_{71} \mathrm{BM}(1: 1.2)$ solar cells can be found in Figure $5 \mathrm{a}, \mathrm{b}$, and their PV performance data are summarized in Table 2, together with those of some structural analogues from literature. PV 

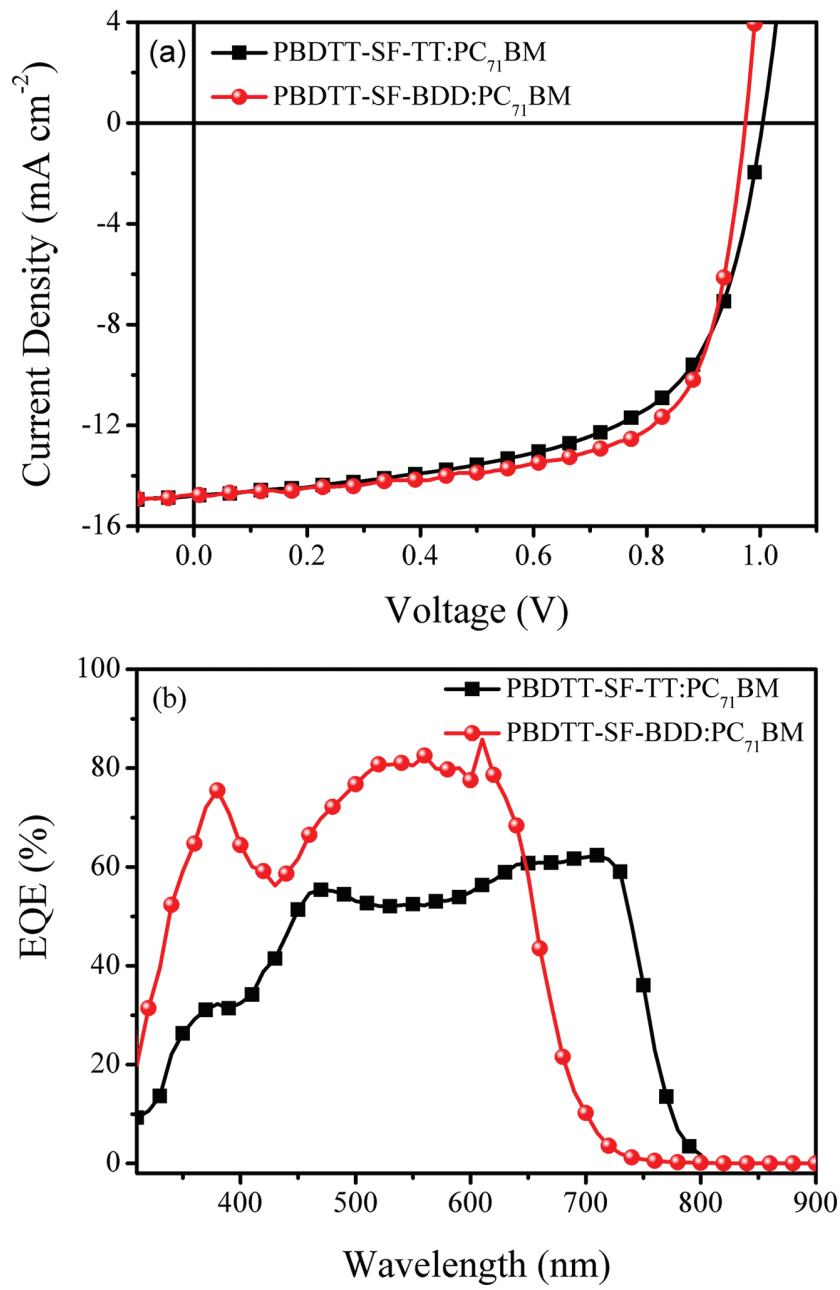

Figure 5. a) $J-V$ curves of PBDTT-SF-TT: $P C_{71} B M$ and PBDTT-SF-BDD: $\mathrm{PC}_{71} \mathrm{BM}$. b) EQE curves of PBDTT-SF-TT: $P C_{71} B M$ and PBDTT-SF-BDD: $\mathrm{PC}_{71} \mathrm{BM}$.

data for solar cells with different blending ratios between either PBDTT-SF-TT or PBDTT-SF-BDD with $\mathrm{PC}_{71} \mathrm{BM}$ (with and without additives or thermal-annealing) are listed in Tables S2-S4 and Figure S3 in the Supporting Information. For PBDTT-SF-TT, the best performance was obtained with the mass ratio of 1:1.5 (polymer to $\mathrm{PC}_{71} \mathrm{BM}$, see Table $\mathrm{S} 2$, Supporting Information), with a $V_{\text {oc }}$ of $1.00 \mathrm{~V}$, a $J_{\mathrm{sc}}$ of $14.79 \mathrm{~mA} \mathrm{~cm}{ }^{-2}$, an FF of $61.08 \%$, affording a total power conversion efficiency of $9.07 \%$. Interestingly, despite the PCE of PBDTT-SF-TT: PC $_{71}$ BM solar cells remains at a nearly the same level, a slightly improved $V_{\text {oc }}$ of $1.01 \mathrm{~V}$ and $J_{\mathrm{sc}}$ of $15.17 \mathrm{~mA} \mathrm{~cm}^{-2}$ was obtained, when treated with $3 \% 1$,8-diiodooctane (DIO) as solvent additive. As all found in Table 2, the obtained $J_{\text {sc }}$ values from PBDTT-TT, PBDTT-S-TT, PBT-3F, and PBDTTSF-TT-based devices were comparable (ranging from 14.99 to $15.32 \mathrm{~mA} \mathrm{~cm}^{-2}$, seen Table 2), due to their close optical absorption characteristics and band gaps. This suggests that neither alkylthiolation on BDTT-TT or on BT-3F (without or with $-\mathrm{F}$ already) nor fluorination on alkylthiolated BDTT-S-TT would result in significant improvement on $J_{\mathrm{sc}}$ but $V_{\mathrm{oc}}$. As discussed above, both alkylthiolation on BDTT-TT and BT-3F resulted a deeper HOMO of $0.11 \mathrm{eV}$ (from $-5.30 \mathrm{eV}$ for PBDTT-TT to $-5.41 \mathrm{eV}$ for PBDTT-S-TT) and $0.24 \mathrm{eV}$ (from $-5.20 \mathrm{eV}$ for PBT-3F to $-5.54 \mathrm{eV}$ for PBDTT-SF-TT), while fluorination on alkylated BDTT and alkylthiolated BDTT-S-TT leads to lower HOMO level of $0.25 \mathrm{eV}$ (from $-4.95 \mathrm{eV}$ for PBT-1F ${ }^{[10]}$ to $-5.20 \mathrm{eV}$ for PBT-3F). All these directly reflected $V_{o c}$ improvement (found in Table 2) among these polymer/PCBM devices were: (a) increased from $0.77(0.60)$ to $0.84 \mathrm{~V}$ via alkylthiolation from PBDTT-TT (PBT-1F) to PBDTT-S-TT; (b) elevated from $0.60(0.77)$ to $0.78 \mathrm{~V}$ via fluorination from PBT-1F ${ }^{[10]}$ (PBDTT-TT) to PBT-3F; (c) enhanced from 0.78 or 0.84 to $1.00 \mathrm{~V}$ via respective alkylthiolation on fluorinated BT-3F or fluorination on alkylated BDTT-S-TT, presenting the coordinative effects of introduction of C-F and C-SR bonding and suggesting the great importance of simultaneous fluorination and alkylthiolation on BDTT-TT series OPV polymers for improved PV performance.

For BDT-BDD series of polymers, alkylthiolation on PB1 (also termed as PBDTBDD ${ }^{[37]}$ ) raises its $V_{\text {oc }}$ from 0.90 to $0.94 \mathrm{~V}$ in its polymer/nonfullerene solar cell due to the resulted deeper HOMO level of PB1-S compared to its structural analog PB1.[27] While in our contribution, fluorination on already alkylthiolated PB1-S furthermore improves the $V_{\text {oc }}$ from 0.94 to $0.97 \mathrm{~V}$ in its polymer/PCBM devices. This again clearly demonstrates the synergy of fluorination and alkylthiolation on alternating BDTT donor and nonfluorinated acceptor polymers.

To date, $J-V$ data of PBDTT-SF-TT and PBDTT-SF-BDD evidences a superior balance between very high $V_{o c}$ and reasonably large $J_{\mathrm{sc}}$ among all the reported BDT or TT-based D-A polymers, as illustrated in Figure $1 \mathrm{~b}$ (raw data are found in Table S1, Supporting Information, and references therein), meanwhile reaching high PCEs of $9.07 \%$ for PBDTT-SF-TT and 9.72\% for PBDTT-SF-BDD within the BDT-based PSCs community. Although PBDTTT-H/PC $\mathrm{P}_{71} \mathrm{BM}$, PBDT-TS1/PC $\mathrm{P}_{71} \mathrm{BM}$, and PBDT-TSR/PC ${ }_{71} \mathrm{BM}$ solar cells had respectively attained facially

Table 2. Performance of fullerene-based PBDTT-SF-TT, PBDTT-SF-BDD, and structural analogue polymer solar cells.

\begin{tabular}{|c|c|c|c|c|c|c|c|}
\hline Polymer/acceptor & $\begin{array}{l}\mathrm{D} / \mathrm{A} \\
{[\mathrm{w} / \mathrm{w}]}\end{array}$ & $\begin{array}{l}\text { DIO } \\
{[\%]}\end{array}$ & $\begin{array}{l}V_{o c} \\
{[V]}\end{array}$ & $\begin{array}{c}J_{\mathrm{sc}} \\
{\left[\mathrm{mA} \mathrm{cm}^{-2}\right]}\end{array}$ & $\begin{array}{l}\mathrm{FF} \\
{[\%]}\end{array}$ & $\begin{array}{l}\text { PCE } \\
{[\%]}\end{array}$ & Reference \\
\hline PTB7/PC ${ }_{71}$ BM & $1: 1.5$ & 3 & 0.74 & 14.50 & 68.97 & 7.40 & [23] \\
\hline PBDTT-TT/PC & $1: 1.5$ & 3 & 0.77 & 14.99 & 63.92 & 7.42 & [17] \\
\hline PBDTT-S-TT/PC & $1: 1.5$ & 0 & 0.84 & 15.32 & 65.49 & 8.42 & [17] \\
\hline $\mathrm{PBT}-3 \mathrm{~F} / \mathrm{PC}_{71} \mathrm{BM}$ & $1: 1.5$ & 3 & 0.78 & 15.20 & 72.4 & 8.60 & [10] \\
\hline PBDTT-SF-TT/PC ${ }_{71} B M$ & $1: 1.5$ & 0 & 1.00 & 14.79 & 61.08 & 9.07 & This work \\
\hline PBDTT-SF-BDD/PC & $1: 1.2$ & 0.5 & 0.97 & 14.70 & 67.50 & 9.72 & This work \\
\hline
\end{tabular}


higher PCE of $9.38 \%, 9.48 \%$, and $10.20 \%$, their high energy losses (as $0.93,0.71$, and $0.79 \mathrm{eV}$ ) from most probably the relaxation of intermolecular charge transfer (CT) states and nonradiation decays, ${ }^{[38]}$ limited all of their $V_{\text {oc }}$ to $0.80 \mathrm{~V}$. While our PBDTT-SF-TT/PC $\mathrm{P}_{71} \mathrm{BM}$ device pronounces its very low $E_{\mathrm{Loss}}$ of $0.59 \mathrm{eV}$ only. Detailed investigation of the energy losses of BDT and/or TT-based PSCs have been executed and the results are summarized in Figure 1a, in evidence PBDTT-SF-TT expresses the lowest $E_{\text {Loss }}$ among such BDT and/or TT-based PSCs with PCE $>8.0 \%$. PBDT-PQD2 revealed an $E_{\text {Loss }}$ of $0.54 \mathrm{eV}$ (as the actual lowest among all BDT polymers) and a high $V_{\text {oc }}$ of 1.08 $\mathrm{V} ;{ }^{[8]}$ however, the too low $J_{\mathrm{sc}}$ of $7.3 \mathrm{~mA} \mathrm{~cm}{ }^{-2}$ led to a PCE of just $3.5 \%$, which is not promising for future actual applications.

In order to understand better the origin of the very low $(0.59 \mathrm{eV})$ and moderately low $(0.88 \mathrm{eV}) E_{\text {Loss }}$ of PBDTT-SF-TT and PBDTT-SF-BDD, photoluminescence (PL) and time-resolved photoluminescence (TRPL) besides EQE experiments on these two polymers and their $\mathrm{PC}_{71} \mathrm{BM}$ blends were conducted, as illustrated in Figure S19 and Figure S20 in the Supporting Information. The emission of PBDTT-SF-TT at $810 \mathrm{~nm}$ and that of PBDTT-SF-BDD at $685 \mathrm{~nm}$ as seen in Figure S19 in the Supporting Information were found fully quenched when they were blended with $\mathrm{PC}_{71} \mathrm{BM}$, revealing the effective photoinduced $\mathrm{CT}^{[39]}$ which strongly indicated a sufficient driving force for CT in PBDTT-SF-TT: PC $_{71}$ BM and PBDTT-SF-BDD: PC $_{71}$ BM blends. Meanwhile, in the film of PBDTT-SF-TT: $\mathrm{PC}_{71} \mathrm{BM}$ blend, the distinct peak found at $\approx 820 \mathrm{~nm}$ could be attributed into the radiative decay of CT states at the interface between the donor and acceptor. $^{[6,40]}$ Such CT states had been also evidenced in other polymer: PCBM and polymer: polymer mixtures previously. ${ }^{[41,42]}$ Beside steady-state PL study, TRPL kinetics of neat polymer PBDTT-SF-TT and PBDTT-SF-BDD and their polymer: $\mathrm{PC}_{71} \mathrm{BM}$ blends had been also investigated for furthermore analyzing the charge photogeneration process in the active layer materials, as illustrated in Figure S20 in the Supporting Information. Well pronounced shorter PL lifetime from 290 ps for PBDTT-SFTT and 230 ps for PBDTT-SF-BDD to 275 and 190 ps for their respective polymer: $\mathrm{PC}_{71} \mathrm{BM}$ blends again strongly proved the efficient charger transfer and separation from donor to acceptor phases. Such found fast rate of CT state formation in PBDTTSF-TT: PC $_{71} \mathrm{BM}$ and PBDTT-SF-BDD: $\mathrm{PC}_{71} \mathrm{BM}$ blends revealed enough driving force for the CT state generation and resulted exciton dissociations, ${ }^{[6,43]}$ also implying higher $E_{\mathrm{CT}}$ which results in lower $E_{\text {Loss }}$ based on the defined $E_{\text {Loss }}=E_{\mathrm{CT}}-e V_{\text {oc }}{ }^{\left[{ }^{6]}\right.}$ On the other hand, such photoenergy loss can be actually beneficial for charge separation and therefore enhance the efficiency of conversion from photons into free charge carries, meaning that changes on $E_{\text {Loss }}$ actually follow the changes on EQE. Thus EQE measurement as seen in Figure $5 \mathrm{~b}$ shows a maximum performance of 0.60 for PBDTT-SF-TT and 0.82 for PBDTT-SF-BDD. This corresponds to the low $E_{\mathrm{Loss}}$ of 0.59 and $0.88 \mathrm{eV}$, respectively, and follows well the trend of an enhanced EQE with larger $E_{\text {Loss }}$ as reported in the pioneer work by Janssen and co-workers..$^{[5,44]}$

In particular, the above-stated remarkably high $V_{\text {oc }}$ of $1.01 \mathrm{~V}$ (Table S2, Supporting Information) obtained for the 3\% DIO annealed solar cell with PBDTT-SF-TT/ $\mathrm{PC}_{71} \mathrm{BM}$ as active layer material should benefit from its distinctly low-lying HOMO energy level that was caused by the further fluorination on BDT units as discussed above. This is found as the main reason that the performance of PBDTT-SF-TT is superior to its structural analogues such as PBDTT-S-TT and PBDTT-TT reported by Li etal., PBDT-3F by Hou etal., and PTB7 by Yu etal. (found in Table2), having a PCE of $8.42 \%$ and $7.42 \%, 8.60 \%$ and $7.40 \%$, which are all noticeably lower than the value reported in this work. This must be owing to either the fluorination or alkylthiolation effects on BDT motif due to such a sole structural difference between PBDTT-SFTT and PBDTT-S-TT or between PBDTT-SF-TT and PBDT-3F, actually meaning the coordinative work of both the two effects. To the best of our knowledge, this is one of the not that many highest $V_{\text {oc }}$ value among all the BDT/TT-based donor-acceptor polymers, and one of the highest $V_{\text {oc }}$ values reported to date for high-performance polymer/PCBM-based BHJ-type PSCs. ${ }^{[45]}$ Another remarkable point is that, as seen in Table 2, Li et al., Hou et al., and Yu et al. have also found an optimal D: A ratio of 1:1.5 for their BDT-based analogue polymers, which is consistent with the findings of this work. In addition, this polymer is insensitive to the weight ratio of donor and acceptor, which is beneficial to practical applications.

For PBDTT-SF-BDD: PC $_{71} \mathrm{BM}$ solar cells, an optimized mass ratio of 1:1.2 was determined, having a $V_{\text {oc }}$ of $0.97 \mathrm{~V}$ combined with a $J_{\mathrm{sc}}$ of $14.70 \mathrm{~mA} \mathrm{~cm}-2$, and an $\mathrm{FF}$ of $67.50 \%$, resulting in a PCE of $9.72 \%$. This remarkably high efficiency and $9.07 \%$ for PBDTT-SF-TT are especially interesting when considering their relatively narrow absorbance spectra (compared with those of representative low band-gap (LBG) polymers) and the band gaps of 1.85 and $1.59 \mathrm{eV}$, respectively. Again the EQE profile (Figure $5 b$ ) presents the onsets of $\approx 790 \mathrm{~nm}$ for PBDT-SFTT and $700 \mathrm{~nm}$ for PBDT-SF-BDD, falling in line with their absorption spectra (Figure 2). This shows that, despite their moderately low $(1.59 \mathrm{eV})$ and wide $(1.85 \mathrm{eV})$ bandgap, the morphology and charge separation of the polymers are of utmost importance for their devices performance as presented below.

\subsection{Additive-Free Property of PBDTT-SF-TT/PC ${ }_{71}$ BM Device}

For optimizing the use of solvent additives (DIO and/or chloronapthalene $(\mathrm{CN})$ ), as presented in Table S3 in the Supporting Information, $3 \%, 5 \%$, and $7 \%$ of DIO, $3 \%$ of $\mathrm{CN}$, and a mixture of $2 \%$ DIO and $3 \% \mathrm{CN}$ have been applied in PBDTT-SF$\mathrm{TT} / \mathrm{PC}_{71} \mathrm{BM}$ device, it is explicitly seen that: (i) $V_{\text {oc }}$ remains a constant at a high value of $1.00 \pm 0.01 \mathrm{~V}$; (ii) $J_{\mathrm{sc}}$ ranges at $14.52 \pm 0.86 \mathrm{~mA} \mathrm{~cm}^{-2}$; (iii) FF falls in line with $0.602 \pm 0.027$; (iv) the hole mobility $\left(\mu_{\mathrm{h}}\right)$ of $1.60 \times 10^{-4} \mathrm{~cm}^{2} \mathrm{~V}^{-1} \mathrm{~s}^{-1}$ without DIO and $1.42 \times 10^{-4} \mathrm{~cm}^{2} \mathrm{~V}^{-1} \mathrm{~s}^{-1}$ with $5 \%$ DIO (found in Figure S5, Supporting Information); (v) therefore final PCE stays at value of $8.50 \pm 0.47 \%$. Such results clearly evidence that DIO and/or $\mathrm{CN}$ additive has no effect on $V_{\mathrm{oc}}$ and very little impact on $J_{\mathrm{sc}}$, FF, and hole mobility. Neither behaved PBDTT-SF-BDD, when seeing the $\mu_{\mathrm{h}}$ values of $1.65 \times 10^{-4}$ or $2.16 \times 10^{-4} \mathrm{~cm}^{2} \mathrm{~V}^{-1} \mathrm{~s}^{-1}$, for film without and with $0.5 \%$ of DIO respectively. Such results appear contradictory to commonly agreed conclusions that the use of solvent additives can indeed noticeably improve both the hole mobility and the $J_{\mathrm{sc}}$, therefore obtain increased PCEs, ${ }^{[46-52]}$ for example, as declared by Jeng and co-workers, the $3 \%$ introduction of DIO or 1-naphtalenethiol in PTB7/PC ${ }_{71} \mathrm{BM}$ solar cell resulted in twice or three times higher $\mu_{\mathrm{h}}$ values (from $9.12 \times 10^{-5}$ to $1.95 \times 10^{-4}$ or $\left.2.75 \times 10^{-4} \mathrm{~cm}^{2} \mathrm{~V}^{-1} \mathrm{~s}^{-1}\right) \cdot{ }^{[53]}$ In a very 
recent study reported by Ding and co-workers, identical optical adsorption, $I-V$ characteristics, and EQE performance among the devices from their homemade polymer PThBDTP: $\mathrm{PC}_{71} \mathrm{BM}$ varying from $1 \%$ to $7 \%$ of DIO has been pronounced, ${ }^{[54]}$ together with comparable film morphologies, which is to large extent in agreement with this work.

The influence of additives on surface morphology and thin film structure of PBDTT-SF-TT/PC ${ }_{71} \mathrm{BM}$ and PBDTT-SF$\mathrm{BDD} / \mathrm{PC}_{71} \mathrm{BM}$ blends had been pursued by means of atomic force microscopy (AFM) (Figure 6) and transmission electron microscope (TEM) (Figure S6, Supporting Information). Figure 6a-d presents the topographic (height) and phase AFM images, showing no large domains observed and revealing possibly good phase separation in nanometer scale between PBDTTSF-TT and $\mathrm{PC}_{71} \mathrm{BM}$. This has been, in angstrom scale, proven by Grazing incidence wide-angle X-ray scattering (GIWAXS) studies, showing that PBDTT-SF-TT is totally X-ray amorphous in the thin film of either its own or when being blended with $\mathrm{PC}_{71} \mathrm{BM}$ (shown in Figure S4, Supporting Information). This is also strongly reflected by such additive independency on their hole mobilities. Again referring to the above mentioned work by Jeng and co-workers, their GIWAXS study on the thin film of TB7/PC ${ }_{71} \mathrm{BM}$ showing an increase of the polymer crystallinity observed which were induced by the DIO or 1-naphtalenethiol additives. ${ }^{[53]}$ This further expresses the distinct additive-inert properties caused by the loss of orderness of PBDTT-SF-TT in angstrom scale. When blending with $\mathrm{PC}_{71} \mathrm{BM}$, the low rootmean-square (RMS) roughness with $0 \%$ and $5 \%$ DIO were found as 0.99 and $2.37 \mathrm{~nm}$ for PBDTT-SF-TT and the RMS with $0 \%$ and $0.5 \%$ DIO were found to be 0.88 and $0.83 \mathrm{~nm}$ for PBDTTSF-BDD, demonstrating a very slight surface fluctuation when having DIO involved. TEM pictures (Figure S6, Supporting
Information) also prove that all PBDTT-SF-TT/PC ${ }_{71} \mathrm{BM}$ films (with/without DIO before) are uniform without large aggregates: the dark region representing $\mathrm{PC}_{71} \mathrm{BM}$ phases (based on their higher electron density) are evenly distributed in the bright area corresponding the polymer phase, indicating very limiting influences on the thin film morphology induced by DIO. All these results denote ideal miscibility between PBDTSF-TT and $\mathrm{PC}_{71} \mathrm{BM}$, which is just the right reason for the independency of DIO additives on $J_{\mathrm{sc}}$, based on such similar surface and bulk morphology of the thin films, comparable charge separation at the polymer-fullerene interface and charge transport in domains are expected. Combination of GIWAXS and AFM study proved their minor variations on FF values in the PV devices from different amount of additives (see Table S3, Supporting Information). Our results sparkle the possible future for fabrication of additive-free BDT-based PSCs.

\section{Conclusion}

Based on the superior and synthetically economic route of the key monomer 4,8-bis (5'-((2"'-ethylhexyl)thio)-4'-fluorothiophen-2'-yl) benzo[1,2-b:4,5- $\left.b^{\prime}\right]$ dithiophene-2,6-diyl)-bis(trimethylstannane) (M1, BDTT-SF) proven by ${ }^{1} \mathrm{H}$ NMR, ${ }^{13} \mathrm{C}$ NMR, and MALDI-TOF MS studies, two new simultaneously fluorinated and alkylthiolated BDT-based donor-acceptor polymers, namely PBDTT-SFTT and PBDTT-SF-BDD, have been designed and successfully synthesized via Stille coupling reactions with yielded moderately high molecular weights and optical band gaps of 1.59 and 1.85 $\mathrm{eV}$, with deep HOMO level of -5.54 and $-5.61 \mathrm{eV}$, respectively. $\mathrm{PC}_{71} \mathrm{BM}$-based $\mathrm{BHJ}$ solar cells pronounce a high PCE of $9.07 \%$ for PBDTT-SF-TT and PCE of $9.72 \%$ for PBDTT-SF-BDD after

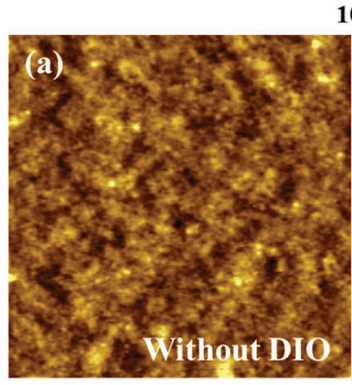

PBDTT-SF-TT:PC ${ }_{71} \mathrm{BM}$
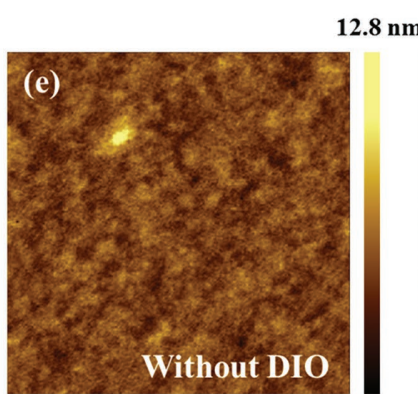

PBDTT-SF-BDD:PC ${ }_{71} \mathrm{BM}^{0}$
$10 \mathrm{~nm}$

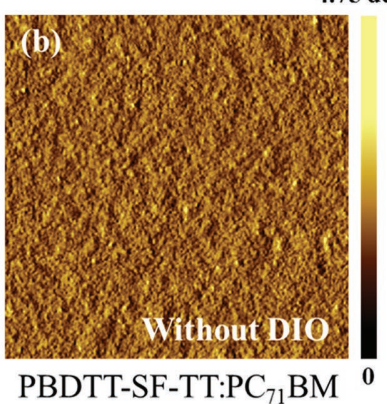

$4.75 \mathrm{deg}$

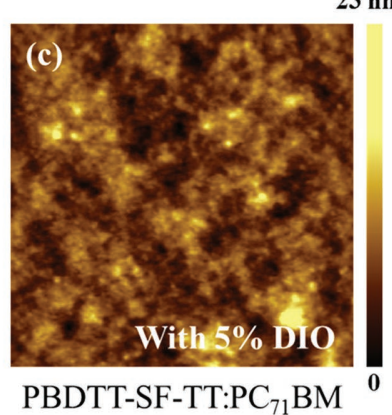

$23 \mathrm{~nm}$
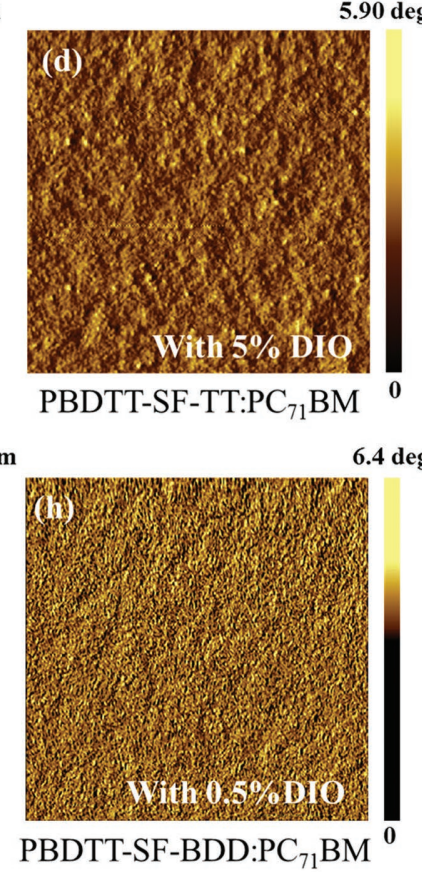

Figure 6. Morphology studies on thin films of PBDT-SF-TT: PC ${ }_{71} B M$ and PBDTT-SF-BDD: PC 71 BM: a,e) AFM height image without DIO, b,f) AFM phase image without DIO, c,g) AFM height image with DIO, d,h) AFM phase image with DIO. The size of the images is $4 \mu \mathrm{m} \times 4 \mu \mathrm{m}$. 
device optimization, respectively. PBDTT-SF-TT presents low energy loss of $0.59 \mathrm{eV}$, which leads to a high $V_{\text {oc }}$ of over $1.00 \mathrm{~V}$. More importantly in both fullerene-based PBDTT-SF-TT and PBDTT-SF-BDD PSCs, high $V_{\text {oc }}$ of 1.01 and $0.97 \mathrm{~V}$ and high $J_{\text {sc }}$ of 15.17 and $14.70 \mathrm{~mA} \mathrm{~cm}{ }^{-2}$ were obtained, respectively, indicating the synergistic effect of fluorination and alkylthiolation on BDT moiety, thus favorable balance between high $V_{\text {oc }}$ and large $J_{\mathrm{sc}}$ that is fundamentally crucial for high OPV performance has been realized in this work. Meanwhile PBDTT-SF-TT, have been confirmed as additive-free PSCs, which is very beneficial to practical applications.

\section{Experimental Section}

Materials: All materials were used as received without further purification, unless otherwise specified. 3-Fluorothiophene and 2'-ethylhexyl-4,6-dibromo-3-fluorothieno[3,4-b]thiophene-2-carboxylate (TT, M2) (Figure S7, Supporting Information) were purchased from Tokyo chemical industry $(\mathrm{TCl})$ and Suna Tech Inc., respectively. The monomer 1,3-bis (5-bromothiophen-2-yl)-5, 7-bis (2-ethylhexyl) benzo[1,2-c:4,5-c'] dithiophene-4,8-dione (BDD) (Figure S8, Supporting Information) and PFN were obtained from Shenzhen Derthon Optoelectronic Materials Science and Technology Co., Ltd. (China). PEDOT: PSS (PH4083) was purchased from Heraeus. The other chemical reagents and dry solvents were obtained from Sigma-Aldrich.

Measurements: ${ }^{1} \mathrm{H}$ NMR and ${ }^{13} \mathrm{C}$ NMR spectra were recorded on a Bruker Avance III $600 \mathrm{MHz}$ spectrometer. UV-vis absorption spectra were carried out on a Varian Cary 50 UV-vis spectrophotometer. TGA was performed on a Perkin-Elmer STA 6000 at a heating rate of $10{ }^{\circ} \mathrm{C} \mathrm{min}^{-1}$ under nitrogen. High-resolution mass spectra were performed on a MALDI-TOF MS. Transmission electron microscopy (TEM) images of the thin films were acquired using a HITACHI $\mathrm{H}-7650$ electron microscope operating at an acceleration voltage of $100 \mathrm{kV}$. AFM images were acquired with Agilent 5400 scanning probe microscope with a Nanodrive controller in tapping mode with MikroMasch NSC-15 AFM tips with resonant frequencies $\approx 300 \mathrm{kHz}$. The SEC measurements were conducted on an Agilent 1260 infinity system using polystyrene as a standard and $\mathrm{CHCl}_{3}$ as an eluent. $\mathrm{CV}$ was measured on a $\mathrm{CHI}$ 660D electrochemical workstation at room temperature in a $0.1 \mathrm{~m}$ solution of tetrabutylammonium perchlorateacetonitrile as the electrolyte with a scan rate of $50 \mathrm{mV} \mathrm{s}^{-1}$. The threeelectrode system consisted of a glassy carbon electrode coated with the polymer film as the working electrode, a platinum wire as the counter electrode and a saturated calomel as the reference electrode. The potential of the ferrocene/ferrocenium redox couple $\left(\mathrm{Fc} / \mathrm{Fc}^{+}\right)$was $-4.80 \mathrm{eV}$ versus the vacuum level, ${ }^{[55]}$ however, had been calibrated with a value of $0.39 \mathrm{~V}$ in this study. The HOMO and the LUMO were estimated by the equation: $\mathrm{HOMO}=-e\left(E_{\mathrm{ox}}^{\mathrm{onset}}-E_{\mathrm{Fc} / \mathrm{Fc}+}+4.80\right)$. The DFT calculations were carried out by using the B3LYP/6-31C basis set (Gaussian 09). GIWAXS patterns were acquired by beamline BL16B1 (Shanghai Synchrotron Radiation Facility). Steady-state PL and TRPL of the spin-coated films based on optimized condition of the solar cells were recorded on an Edinburgh Instruments FLS 980 threemonochromator fluorescence spectrometer in ambient atmosphere with excitation using $375 \mathrm{~nm}$ laser.

Solar Cells Fabrication and Characterization: Photovoltaic devices

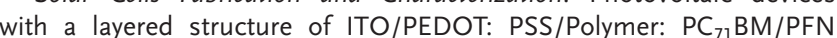
$(2 \mathrm{~nm}) / \mathrm{Al}(100 \mathrm{~nm})$ were fabricated on ITO coated glass substrates $(15 \mathrm{~mm} \times 15 \mathrm{~mm})$. The ITO coated glasses were cleaned in an ultrasonic bath with detergent, ultrapure water, acetone, and isopropyl alcohol sequentially for $20 \mathrm{~min}$, and then oxygen plasma treated for $6 \mathrm{~min}$. The substrates were spin coated with PEDOT: PSS at $5000 \mathrm{rpm}$, and dried at $150{ }^{\circ} \mathrm{C}$ for $20 \mathrm{~min}$ in air. The polymer and $\mathrm{PC}_{71} \mathrm{BM}$ were dissolved in 0 -DCB with fixed polymer concentration of $10 \mathrm{mg} \mathrm{mL}^{-1}$, and stirred overnight in glovebox. And then the blend solution was spin coated on
PEDOT: PSS modified ITO substrates resulting thickness of the active layer around $97 \pm 5 \mathrm{~nm}$. Subsequently, an ultrathin layer of PFN with a $0.2 \mathrm{mg} \mathrm{mL}^{-1} \mathrm{PFN}$ in methanol was casted on the active layer under $2600 \mathrm{rpm}$ for $20 \mathrm{~s}$. Finally, Al $(100 \mathrm{~nm})$ were thermally evaporated in a vacuum of $2 \times 10^{-4} \mathrm{~Pa}$ on top of the PFN layer as cathode. Photovoltaic performance was characterized under illumination with an AM 1.5C $\left(100 \mathrm{~mW} \mathrm{~cm}{ }^{-2}\right)$, and the current-voltage curves were recorded using a Keithley 2400 source meter. The EQE of solar cells were analyzed using a certified Newport incident photon conversion efficiency measurement system.

The hole mobility was measured using the space-charge-limited current (SCLC) method with a device architecture of ITO/PEDOT: PSS/ active layer $/ \mathrm{MoO}_{3} / \mathrm{Al}$. The SCLC is described by the equation $J_{\mathrm{SCLC}}=$ $(9 / 8) \varepsilon_{\mathrm{o}} \varepsilon_{\mathrm{r}} \mu_{\mathrm{cc}}\left(\left(V^{2}\right) /\left(L^{3}\right)\right)$, whereas $J_{\mathrm{SCLC}}$ is the current density, $\varepsilon_{\mathrm{o}}$ is the permittivity of free space $\left(8.85 \times 10^{-12} \mathrm{~F} \mathrm{~m}^{-1}\right), \varepsilon_{\mathrm{r}}$ is the relative dielectric constant of the transport medium, $\mu_{\mathrm{cc}}$ is the free charge carrier mobility (either hole or electron mobility), $V$ is the internal potential in the device, and $L$ is the thickness of the active layer (measured by a profilometer).

Synthesis: Lithium diisopropylamide (LDA): In a $100 \mathrm{~mL}$ nitrogen purged three-neck flask, $n$-BuLi ( $15 \mathrm{~mL}, 25.5 \mathrm{mmol}, 2.5 \mathrm{~m}$ in hexane) was added dropwise into a solution of diisopropylamine $(3.56 \mathrm{~mL}$, $25.5 \mathrm{mmol})$ in anhydrous THF $(13 \mathrm{~mL})$ at $-78{ }^{\circ} \mathrm{C}$ under a nitrogen atmosphere. The resulting mixture was stirred at $-78^{\circ} \mathrm{C}$ for $20 \mathrm{~min}$ and then slowly became off-white suspension. Subsequently, the solution was slowly warmed up to $0{ }^{\circ} \mathrm{C}$ and stirred for $15 \mathrm{~min}$. After stirring at room temperature for $5 \mathrm{~min}$, the freshly prepared LDA was obtained as a clear solution and directly used in next steps.

2-(2'-Ethylhexyl)thio-3-fluorothiophene (2): 3-Fluorothiophene $(2.0 \mathrm{~g}$, $19.58 \mathrm{mmol})$ and anhydrous tetrahydrofuran (THF) $(40 \mathrm{~mL})$ were added into a well-dried $250 \mathrm{~mL}$ three-neck flask under nitrogen protection. The mixture was cooled to $-78{ }^{\circ} \mathrm{C}$ and the freshly prepared LDA solution $(19.7 \mathrm{mmol})$ was added dropwise. The resulting solution was then slowly warmed up to $0{ }^{\circ} \mathrm{C}$ and stirred for an additional $1 \mathrm{~h}$. After that, sulfur powder $(0.63 \mathrm{~g}, 19.5 \mathrm{mmol})$ was quickly added in one portion, and then the resulting suspension was stirred at $0{ }^{\circ} \mathrm{C}$ for $2 \mathrm{~h}$. Subsequently, 2-ethylhexylbromide (3.76 g, $19.4 \mathrm{mmol})$ were simultaneously fed for an overnight reaction at ambient temperature under nitrogen atmosphere. $\mathrm{NH}_{4} \mathrm{Cl}$ ice-water solution was used for quenching the reaction, followed by ether-extraction with furthermore water washing and drying under $\mathrm{MgSO}_{4}$. Final silica gel column chromatography using hexane as the eluent afforded colorless oil as the final product, compound $2(3.11 \mathrm{~g}$, yield $65 \%)$. ${ }^{1} \mathrm{H}$ NMR $\left(600 \mathrm{MHz}, \mathrm{CDCl}_{3}\right): \delta(\mathrm{ppm}) 7.20(\mathrm{dd}, 1 \mathrm{H}), 6.79$ $(\mathrm{dd}, 1 \mathrm{H}), 2.74(\mathrm{~m}, 2 \mathrm{H}), 1.51-1.20(\mathrm{~m}, 9 \mathrm{H}), 0.88(\mathrm{t}, 3 \mathrm{H}), 0.85(\mathrm{t}, 3 \mathrm{H})$ (Figure S9, Supporting Information). ${ }^{13} \mathrm{C}$ NMR (150 MHz, CDCl3): $\delta$ (ppm) 160.36, 158.63, 126.70, 126.64, 117.69, 117.51, 113.35, 113.22, $42.88,39.23,32.04,28.82,25.26,23.05,14.21,10.78$ (Figure S10, Supporting Information)

4, 8-Bis (5'-((2"-ethylhexyl)thio)-4'-fluorothiophen-2'-yl) benzo[1,2$b: 4,5-b^{\prime}$ ]dithiophene (3): In a $250 \mathrm{~mL}$ nitrogen purged three-neck flask, the freshly prepared LDA solution $(12.5 \mathrm{mmol})$ was added dropwise into an anhydrous THF solution $(25 \mathrm{~mL})$ of 2 -(2'-ethylhexyl)thio-3fluorothiophene (3) $(3.0 \mathrm{~g}, 12.2 \mathrm{mmol})$ at $-78{ }^{\circ} \mathrm{C}$ under nitrogen protection. The mixture was stirred for $1.5 \mathrm{~h}$, and then benzo[1,2b:4,5- $b^{\prime}$ ]dithiophene-4,8-dione $(0.91 \mathrm{~g}, 4 \mathrm{mmol})$ was added in one portion and again stirred for $3 \mathrm{~h}$ at $-78{ }^{\circ} \mathrm{C}$, then followed by slowly warming up to room temperature for $12 \mathrm{~h}$, and was finally reacted at $55^{\circ} \mathrm{C}$ for an additional $2.0 \mathrm{~h}$. Subsequently, the mixture was cooled down to room temperature and Tin (II) chloride dihydrate $(6.48 \mathrm{~g}, 28.7 \mathrm{mmol})$ in $10 \% \mathrm{HCl}(15 \mathrm{~mL})$ was added and then the resulting solution was stirred for an additional $2.5 \mathrm{~h}$ at $55^{\circ} \mathrm{C}$. The reaction was quenched with deionized water $(50 \mathrm{~mL})$ and then extracted three times with diethyl ether. The combined organic phase was dried with anhydrous $\mathrm{Na}_{2} \mathrm{SO}_{4}$ and then concentrated by rotary evaporator. The residue was purified via a silica gel column chromatography using hexane as the eluent to obtain compound 3 as a bright-yellow sticky liquid $(2.47 \mathrm{~g}, 91 \%$ yield). ${ }^{1} \mathrm{H}$ NMR $\left(600 \mathrm{MHz}, \mathrm{CDCl}_{3}\right): \delta(\mathrm{ppm}) 7.61(\mathrm{~d}, 2 \mathrm{H}), 7.52(\mathrm{~d}, 2 \mathrm{H}), 7.20$ $(\mathrm{s}, 2 \mathrm{H}), 2.88(\mathrm{~m}, 4 \mathrm{H}), 1.60(\mathrm{~m}, 2 \mathrm{H}), 1.56-1.39(\mathrm{~m}, 8 \mathrm{H}), 1.34-1.27(\mathrm{~m}$, $8 \mathrm{H}), 0.91(\mathrm{t}, 12 \mathrm{H})$ (Figure S11, Supporting Information). ${ }^{13} \mathrm{C}$ NMR 
(150 MHz, $\mathrm{CDCl} 3): \delta$ (ppm) 159.70, 157.97, 139.75, 139.69, 139.09, $136.54,128.64,123.55,122.89,118.45,118.27,114.89,114.85,42.91$, $39.49,32.17,28.93,25.40,23.07,14.27,10.92$ (Figure S12, Supporting Information).

4, 8-Bis (5'-((2"'-ethylhexyl)thio)-4'-fluorothiophen-2'-yl) benzo[1,2$b: 4,5-b^{\prime}$ ]dithiophene-2, 6-dipl)-bis(trimethy/stannane) (M1): In a $250 \mathrm{~mL}$ flame-dried flask, $n$-BuLi ( $2.2 \mathrm{~mL}, 5.5 \mathrm{mmol}, 2.5 \mathrm{~m}$ in hexane) was slowly added dropwise to a dry THF solution $(60 \mathrm{~mL})$ of compound $3(1.54 \mathrm{~g}$, $2.27 \mathrm{mmol}$ ) at $-78{ }^{\circ} \mathrm{C}$ under argon protection. The solution was stirred at $-78{ }^{\circ} \mathrm{C}$ for $1.5 \mathrm{~h}$ and then trimethyltin chloride solution $(6.6 \mathrm{~mL}$, $6.6 \mathrm{mmol}, 1.0 \mathrm{M}$ in THF) was added in one portion via a syringe at $-78^{\circ} \mathrm{C}$. The resulting mixture was stirred for $1 \mathrm{~h}$ at this temperature and then slowly warmed up to room temperature. After stirring overnight, the solution was poured into cold deionized water $(100 \mathrm{~mL})$ and extracted with diethyl ether three times. The combined organic layer was dried over anhydrous $\mathrm{Na}_{2} \mathrm{SO}_{4}$. The solvent was removed via rotary evaporation, and the crude product was recrystallized from isopropano to afford a yellow powder monomer M1 (1.56 g, 68\% yield). ${ }^{1} \mathrm{H}$ NMR $\left(600 \mathrm{MHz}, \mathrm{CDCl}_{3}\right): \delta(\mathrm{ppm}) 7.63(\mathrm{~s}, 2 \mathrm{H}), 7.21(\mathrm{~s}, 2 \mathrm{H}), 2.89(\mathrm{~m}, 4 \mathrm{H}), 1.62$ $(\mathrm{m}, 2 \mathrm{H}), 1.56-1.39(\mathrm{~m}, 8 \mathrm{H}), 1.34-1.27(\mathrm{~m}, 8 \mathrm{H}), 0.92(\mathrm{~m}, 12 \mathrm{H}), 0.42$ (s, 18H) (Figure S13, Supporting Information). ${ }^{13} \mathrm{C} \mathrm{NMR}(150 \mathrm{MHz}$, $\mathrm{CDCl} 3$ ): $\delta$ (ppm) 159.74, 158.01, 144.11, 143.38, 140.66, 140.60, 137.32, $130.37,121.80,118.27,118.09,114.36,114.23,42.89,39.49,32.16$, $28.91,25.40,23.07,14.29,10.96,-8.12$ (Figure S14, Supporting Information). Molecular weight calcd. for $\mathrm{C}_{40} \mathrm{H}_{56} \mathrm{~F}_{2} \mathrm{~S}_{6} \mathrm{Sn}_{2}: \mathrm{m} / \mathrm{z}=1004.7$; MALDI-TOF MS found: 1004.1.

Polymerization for PBDTT-SF-TT: To a well-dried $50 \mathrm{~mL}$ round bottom flask was added M1 $(0.3517 \mathrm{~g}, 0.35 \mathrm{mmol}), \mathrm{M} 2(0.1653 \mathrm{~g}, 0.35 \mathrm{mmol})$, $\mathrm{Pd}\left(\mathrm{PPh}_{3}\right)_{4}(23 \mathrm{mg}, 0.02 \mathrm{mmol})$, anhydrous toluene $(12 \mathrm{~mL})$, and anhydrous DMF $(2.4 \mathrm{~mL})$ under nitrogen. The mixture was then stirred and degassed three times by using nitrogen at $-78^{\circ} \mathrm{C}$. After removing the dry-ice bath, the solution was heated to $110^{\circ} \mathrm{C}$ and stirred for $40 \mathrm{~h}$ under nitrogen protection. The solution slowly became dark blue jelly like substance as it was cooled down to room temperature (Figure S17, Supporting Information). Subsequently, $25 \mathrm{~mL}$ methanol was added and then the polymers were collected by filtration. For further purification, the crude polymer was subjected to Soxhlet extraction respectively with methanol, acetone, hexane, and $\mathrm{CHCl}_{3}$ to remove oligomers and catalyst. The residue was dissolved in $0-\mathrm{DCB}$ at $100^{\circ} \mathrm{C}$ and then purified by using a flash chromatography on silica gel (80-100 mesh) flushed with $o$-DCB. The polymer was precipitated from methanol as a dark solid dried under vacuum for $40 \mathrm{~h}$. The ${ }^{1} \mathrm{H}$ NMR spectra of the target polymer was given in Figure S15 in the Supporting Information. Yield: $75 \%$. $M_{\mathrm{n}}=58127, \mathrm{PDI}=2.53$.

Polymerization for PBDTT-SF-BDD: Monomer M1 $(0.3517 \mathrm{~g}$, $0.35 \mathrm{mmol}), \operatorname{BDD}(0.2684 \mathrm{~g}, 0.35 \mathrm{mmol}), \mathrm{Pd}\left(\mathrm{PPh}_{3}\right)_{4}(23 \mathrm{mg}, 0.02 \mathrm{mmol}$ ), and anhydrous toluene $(15 \mathrm{~mL})$ were stirred and degassed four times at $-78{ }^{\circ} \mathrm{C}$. The solution was slowly heated up to $110^{\circ} \mathrm{C}$ and stirred for $3 \mathrm{~h}$ under nitrogen protection. During the polymerization process, the color of the solution was changed from orange to dark red (Figure S18, Supporting Information). The solution slowly became jelly like substance when cooled down to room temperature. Subsequently, $25 \mathrm{~mL}$ methanol was added and then the polymer was collected by filtration (Figure S18, Supporting Information). For further purification, the crude polymer was washed by Soxhlet extraction with methanol, acetone, hexane, dichloromethane $\left(\mathrm{CH}_{2} \mathrm{Cl}_{2}\right)$ and $\mathrm{CHCl}_{3}$ to remove oligomers and catalyst. The residue was dissolved in $0-\mathrm{DCB}$ at $100{ }^{\circ} \mathrm{C}$ and then purified by using a flash chromatography on silica gel (80-100 mesh) flushed with $o$-DCB. When concentrated by rotary evaporator, the target polymer was obtained as a dark solid after drying under vacuum for $40 \mathrm{~h}$. The ${ }^{1} \mathrm{H}$ NMR spectrum of the target polymer was given in Figure S16 in the Supporting Information. Yield: $70 \% . M_{n}=33358, P D I=3.92$.

\section{Supporting Information}

Supporting Information is available from the Wiley Online Library or from the author.

\section{Acknowledgements}

The authors would like to gratefully acknowledge the financial support from the Danish National Research Foundation (DNRF) for the Danish-Chinese Center for Organic-based Photovoltaic Cells with Morphological Control and the National Natural Science Foundation of China (51573205). X.B. thanks the Youth Innovation Promotion Association CAS (2016194) for financial support. The authors thank beamline BL16B1 (Shanghai Synchrotron Radiation Facility) for providing the beam time. Support from the Sino-Danish Center for Education and Research (SDC) is also fully acknowledged. The authors thank Dr. R. Zhu and Prof. X. Li (China University of Petroleum (East China)) for the measurement of the PL spectra.

\section{Conflict of Interest}

The authors declare no conflict of interest.

\section{Keywords}

alkylthiolation, benzo[1,2-b:4,5- $\left.b^{\prime}\right]$ dithiophene, fluorination, polymer solar cells, synergistic effect

Received: May 29, 2017

Revised: August 27, 2017

Published online:

[1] W. Zhao, D. Qian, S. Zhang, S. Li, O. Inganas, F. Gao, J. Hou, Adv. Mater. 2016, 28, 4734.

[2] Z. Zheng, S. Zhang, J. Zhang, Y. Qin, W. Li, R. Yu, Z. Wei, J. Hou, Adv. Mater. 2016, 28, 5133.

[3] S. Li, L. Ye, W. Zhao, S. Zhang, S. Mukherjee, H. Ade, J. Hou, Adv. Mater. 2016, 28, 9423

[4] W. Zhao, S. Li, H. Yao, S. Zhang, Y. Zhang, B. Yang, J. Hou, J. Am. Chem. Soc. 2017, 139, 7148.

[5] W. Li, K. H. Hendriks, A. Furlan, M. M. Wienk, R. A. J. Janssen, J. Am. Chem. Soc. 2015, 137, 2231.

[6] C. Wang, X. Xu, W. Zhang, J. Bergqvist, Y. Xia, X. Meng, K. Bini, W. Ma, A. Yartsev, K. Vandewal, M. R. Andersson, O. Inganas, M. Fahlman, E. Wang, Adv. Energy Mater. 2016, 6, 1600148.

[7] D. Veldman, S. C. J. Meskers, R. A. J. Janssen, Adv. Funct. Mater. 2009, 19, 1939.

[8] X. Xu, C. Wang, O. Backe, D. I. James, K. Bini, E. Olsson, M. R. Andersson, M. Fahlman, E. Wang, Polym. Chem. 2015, 6, 4624.

[9] H. Yao, H. Zhang, L. Ye, W. Zhao, S. Zhang, J. Hou, ACS Appl. Mater. Interfaces 2016, 8, 3575 .

[10] M. Zhang, X. Guo, S. Zhang, J. Hou, Adv. Mater. 2014, 26, 1118.

[11] L. Ye, S. Zhang, W. Zhao, H. Yao, J. Hou, Chem. Mater. 2014, 26, 3603.

[12] H. Yao, W. Zhao, Z. Zheng, Y. Cui, J. Zhang, Z. Wei, J. Hou, J. Mater. Chem. A 2016, 4, 1708 .

[13] Y.-J. Cheng, J. Luo, S. Huang, X. Zhou, Z. Shi, T.-D. Kim, D. H. Bale, S. Takahashi, A. Yick, B. M. Polishak, S.-H. Jang, L. R. Dalton, P. J. Reid, W. H. Steier, A. K. Y. Jen, Chem. Mater. 2008, 20, 5047

[14] J. Hou, B. Fan, L. Huo, C. He, C. Yang, Y. Li, J. Polym. Sci., Part A: Polym. Chem. 2006, 44, 1279.

[15] D. Lee, S. W. Stone, J. P. Ferraris, Chem. Commun. 2011, 47, 10987.

[16] D. Lee, E. Hubijar, G. J. D. Kalaw, J. P. Ferraris, Chem. Mater. 2012, 24, 2534.

[17] C. Cui, W.-Y. Wong, Y. Li, Energy Environ. Sci. 2014, 7, 2276. 
[18] F. Meyer, Prog. Polym. Sci. 2015, 47, 70

[19] P. Boufflet, Y. Han, Z. Fei, N. D. Treat, R. Li, D.-M. Smilgies, N. Stingelin, T. D. Anthopoulos, M. Heeney, Adv. Funct. Mater. 2015, 25, 7038

[20] H. J. Son, W. Wang, T. Xu, Y. Liang, Y. Wu, G. Li, L. Yu, J. Am. Chem. Soc. 2011, 133, 1885.

[21] B. C. Schroeder, R. S. Ashraf, S. Thomas, A. J. P. White, L. Biniek, C. B. Nielsen, W. Zhang, Z. Huang, P. S. Tuladhar, S. E. Watkins, T. D. Anthopoulos, J. R. Durrant, I. McCulloch, Chem. Commun. 2012, 48, 7699 .

[22] B. C. Schroeder, Z. Huang, R. S. Ashraf, J. Smith, P. D'Angelo, S. E. Watkins, T. D. Anthopoulos, J. R. Durrant, I. McCulloch, Adv. Funct. Mater. 2012, 22, 1663.

[23] Y. Y. Liang, Z. Xu, J. B. Xia, S. T. Tsai, Y. Wu, G. Li, C. Ray, L. P. Yu, Adv. Mater. 2010, 22, E135.

[24] Y. Zhang, J. Zou, C.-C. Cheuh, H.-L. Yip, A. K. Y. Jen, Macromolecules 2012, 45, 5427.

[25] C. Cui, Z. He, Y. Wu, X. Cheng, H. Wu, Y. Li, Y. Cao, W.-Y. Wong, Energy Environ. Sci. 2016, 9, 885.

[26] K. Reichenbacher, H. I. Suss, J. Hulliger, Chem. Soc. Rev. 2005, 34, 22.

[27] Q. Wang, S. Zhang, B. Xu, S. Li, B. Yang, W. Yuan, J. Hou, J. Phys. Chem. C 2017, 121, 4825 .

[28] Y. Liu, J. Zhao, Z. Li, C. Mu, W. Ma, H. Hu, K. Jiang, H. Lin, H. Ade, H. Yan, Nat. Commun. 2014, 5, 1.

[29] W. Yue, X. Huang, J. Yuan, W. Ma, F. C. Krebs, D. Yu, J. Mater. Chem. A 2013, 1, 10116

[30] J. Wang, M. Xiao, W. Chen, M. Qiu, Z. Du, W. Zhu, S. Wen, N. Wang, R. Yang, Macromolecules 2014, 47, 7823.

[31] S. Zhang, Y. Qin, M. A. Uddin, B. Jang, W. Zhao, D. Liu, H. Y. Woo, J. Hou, Macromolecules 2016, 49, 2993.

[32] C.-Y. Kuo, W. Nie, H. Tsai, H.-J. Yen, A. D. Mohite, G. Gupta, A. M. Dattelbaum, D. J. William, K. C. Cha, Y. Yang, L. Wang, H.-L. Wang, Macromolecules 2014, 47, 1008.

[33] Y. Liu, W. Zhao, Y. Wu, J. Zhang, G. Li, W. Li, W. Ma, J. Hou, Z. Bo, J. Mater. Chem. A 2016, 4, 8097

[34] S. Zhang, H. Fan, Y. Liu, G. Zhao, Q. Li, Y. Li, X. Zhan, J. Polym. Sci., Part A: Polym. Chem. 2009, 47, 2843.

[35] S. Li, L. Ye, Q. Wang, S. Zhang, W. Zhao, J. Hou, Org. Electron. 2016, 28, 39
[36] S. B. Darling, M. Sternberg, J. Phys. Chem. B 2009, 113, 6215.

[37] D. P. Qian, L. Ye, M. J. Zhang, Y. R. Liang, L. J. Li, Y. Huang, X. Guo, S. Q. Zhang, Z. A. Tan, J. H. Hou, Macromolecules 2012, 45, 9611.

[38] S. M. Menke, A. Sadhanala, M. Nikolka, N. A. Ran, M. K. Ravva, S. Abdel-Azeim, H. L. Stern, M. Wang, H. Sirringhaus, N. Thuc-Quyen, J.-L. Bredas, G. C. Bazan, R. H. Friend, ACS Nano 2016, 10, 10736

[39] M. H. Tong, N. E. Coates, D. Moses, A. J. Heeger, S. Beaupre, M. Leclerc, Phys. Rev. B 2010, 81, 6 .

[40] Y. Zhou, K. Tvingstedt, F. Zhang, C. Du, W.-X. Ni, M. R. Andersson, O. Inganäs, Adv. Funct. Mater. 2009, 19, 3293.

[41] T. Offermans, P. A. van Hal, S. C. J. Meskers, M. M. Koetse, R. A. J. Janssen, Phys. Rev. B 2005, 72, 11.

[42] Z. Ma, W. Sun, S. Himmelberger, K. Vandewal, Z. Tang, J. Bergqvist, A. Salleo, J. W. Andreasen, O. Inganas, M. R. Andersson, C. Muller, F. Zhang, E. Wang, Energy Environ. Sci. 2014, 7, 361

[43] A. C. Morteani, R. H. Friend, C. Silva, J. Chem. Phys. 2005, 122, 7.

[44] W. W. Li, W. S. C. Roelofs, M. M. Wienk, R. A. J. Janssen, J. Am. Chem. Soc. 2012, 134, 13787.

[45] H. Yao, L. Ye, H. Zhang, S. Li, S. Zhang, J. Hou, Chem. Rev. 2016, 116, 7397.

[46] S. J. Lou, J. M. Szarko, T. Xu, L. Yu, T. J. Marks, L. X. Chen, J. Am. Chem. Soc. 2011, 133, 20661.

[47] M.-S. Su, C.-Y. Kuo, M.-C. Yuan, U. S. Jeng, C.-J. Su, K.-H. Wei, Adv. Mater. 2011, 23, 3315

[48] C. V. Hoven, X. D. Dang, R. C. Coffin, J. Peet, T. Q. Nguyen, G. C. Bazan, Adv. Mater. 2010, 22, E63.

[49] G. Li, R. Zhu, Y. Yang, Nat. Photonics 2012, 6, 153.

[50] H. Li, J. Cao, Q. Zhou, L. Ding, J. Wang, Nano Energy 2015, 15, 125.

[51] J. K. Lee, W. L. Ma, C. J. Brabec, J. Yuen, J. S. Moon, J. Y. Kim, K. Lee, G. C. Bazan, A. J. Heeger, J. Am. Chem. Soc. 2008, 130, 3619.

[52] B. A. Collins, Z. Li, J. R. Tumbleston, E. Gann, C. R. McNeill, H. Ade, Adv. Energy Mater. 2013, 3, 65.

[53] H.-J. Jhuo, S.-H. Liao, Y.-L. Li, P.-N. Yeh, S.-A. Chen, W.-R. Wu, C.-J. Su, J.-J. Lee, N. L. Yamada, U. S. Jeng, Adv. Funct. Mater. 2016, 26, 3094

[54] H. Li, D. He, P. Mao, Y. Wei, L. Ding, J. Wang, Adv. Energy Mater. 2017, https://doi.org/10.1002/aenm.201602663.

[55] J. Pommerehne, H. Vestweber, W. Guss, R. F. Mahrt, H. Bässler, M. Porsch, J. Daub, Adv. Mater. 1995, 7, 551. 